\title{
ESEEM Studies of Substrate Water and Small Alcohol Binding to the Oxygen-Evolving Complex of Photosystem II during Functional Turnover ${ }^{\dagger}$
}

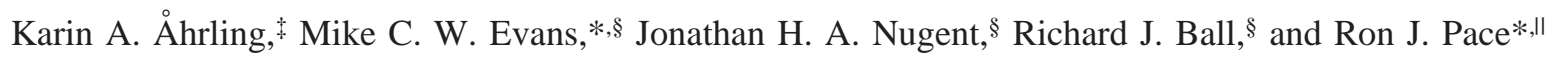 \\ Research School of Biological Science, Australian National University, Canberra, ACT 0200, Australia, Department of Biology, \\ University College London, Gower Street, London WCIE 6BT, U.K., and Department of Chemistry, \\ Faculty of Science, Australian National University, Canberra, ACT 0200, Australia
}

Received October 20, 2005; Revised Manuscript Received April 3, 2006

\begin{abstract}
We report the first examination of exchangeable proton and $\mathrm{MeOH}$ interactions with the $\mathrm{Mn}$ catalytic cluster in photosystem II, under functional flash turnover conditions, using ${ }^{2} \mathrm{H}$ ESEEM spectroscopy on the $\mathrm{S}_{2}$ and $\mathrm{S}_{0}$ multiline states. Deuterium-labeled water $\left(\mathrm{D}_{2} \mathrm{O}\right)$ and methyl $d_{3}$-labeled methanol (DMeOH) are employed. It was discovered that a hyperfine resolved multiline $\mathrm{S}_{0}$ signal could be seen in the presence of $\mathrm{D}_{2} \mathrm{O}$, the hyperfine structure of which depended on the presence or absence of methanol $(\mathrm{MeOH})$. In the presence of $\mathrm{DMeOH}$, significant dipolar coupling of the three methyl deuterons to the multiline centers in the $\mathrm{S}_{2}$ and $\mathrm{S}_{0}$ states was seen $\left(\mathrm{S}_{2}, 0.65,0.39(2) \mathrm{MHz}\right.$; and $\left.\mathrm{S}_{0}, 0.60,0.37(2) \mathrm{MHz}\right)$. These are consistent with direct binding of the methoxy fragment to Mn. Assuming terminal Mn-OMe ligation, the couplings indicated a spin projection coefficient $(\rho)$ magnitude of $\sim 2$ for the ligating $\mathrm{Mn}$ in both the $\mathrm{S}_{2}$ and $\mathrm{S}_{0}$ states, with inferred $\mathrm{Mn}-\mathrm{O}$ distances of $\sim 1.9-2.0 \AA$. In the presence of $\mathrm{D}_{2} \mathrm{O}$, four classes of exchangeable deuterons were identified by ESEEM in $S_{2}$ and $S_{0}$. Three of these classes $(1,2$, and 4) exhibited populations and coupling strengths that were essentially constant under various conditions of sample preparation, illumination turnover, and small alcohol addition. Class 3 could be modeled with constant coupling but a highly variable deuteron population $\left(n_{3} \sim 0-10\right)$ depending in part on the preparation used. For all classes, the coupling parameters were very similar in $\mathrm{S}_{2}$ and $\mathrm{S}_{0}$. The favored interpretation is that the two strongest coupling classes (1 and 2) represent close binding of one water molecule to a single Mn which has an oxidation state of II in $\mathrm{S}_{0}$ and III in $\mathrm{S}_{2}$, and $\rho \sim 2$ in both cases. This water is not displaced by $\mathrm{MeOH}$, but either the water or $\mathrm{MeOH}$ is singly deprotonated upon $\mathrm{MeOH}$ binding. Class 4 represents $\sim 2$ water molecules which are not closely bound to $\mathrm{Mn}(\mathrm{Mn}-$ deuteron distances of $\sim 3.7-4.7 \AA)$. Class 3 probably represents protein matrix protons within $\sim 4 \AA$ of the $\mathrm{Mn}$ in the cluster, which can be variably exchanged in different preparations.
\end{abstract}

The oxidation of water in photosynthesis is catalyzed by a membrane-bound protein-pigment assembly, photosystem II (PSII). ${ }^{1}$ Photo-oxidation of the reaction center chlorophyll pigments, P680, within this photosystem provides the high oxidizing potential necessary for water splitting. The reaction

This work was supported in part by grants from the U.K. Biotechnology and Biological Sciences Research Council. R.J.P. acknowledges support from the Australian Research Council.

* To whom correspondence should be addressed. M.C.W.E.: Department of Biology, Darwin Building, University College London, Gower St, London WC1E 6BT, U.K.; phone, +44-207-679-7312; fax, +442070679-7098; e-mail, mcw.evans@ucl.ac.uk. R.J.P.: Department of Chemistry, Faculty of Science, Australian National University, Canberra, ACT 0200, Australia; phone, +61 26125 4546; fax, +61 26125 8997; e-mail, Ron.Pace@anu.edu.au.

Research School of Biological Science, Australian National University.

$\S$ University College London.

"Department of Chemistry, Faculty of Science, Australian National University.

${ }^{1}$ Abbreviations: EPR, electron paramagnetic resonance; ESEEM, electron spin-echo envelope modulation; ENDOR, electron nuclear double resonance; EXAFS, extended X-ray absorption fine structure; $\mathrm{MeOH}$, methanol; DMeOH, $\mathrm{C}^{2} \mathrm{H}_{3} \mathrm{OH} ; \mathrm{D}_{2} \mathrm{O},{ }^{2} \mathrm{H}_{2} \mathrm{O}$; EtOH, ethanol; PSII, photosystem II; CW, continuous wave; MES, 2-( $N$-morpholino)ethanesulfonic acid; EDTA, ethylenediaminetetraacetic acid; chl, chlorophyll; PpBQ, $p$-phenyl benzoquinone; Cyt, cytochrome. involves a $\mathrm{Mn}_{4} / \mathrm{Ca}$ center involving $\mu$-oxo-bridged $\mathrm{Mn}$ atoms that functions as an oxidant accumulator and is thought to provide the catalytic site for water oxidation. Classical experiments $(1,2)$ show that the evolution of oxygen following single-turnover flash illumination of PSII occurs on the third flash, and subsequently on every fourth flash. This process is formalized in the S state hypothesis. This requires a charge accumulation system that has five formal redox states, conventionally labeled $\mathrm{S}_{0}-\mathrm{S}_{4}$. The dark stable resting state is $S_{1}$. Each turnover of the reaction center advances the oxidation state of the complex from $\mathrm{S}_{0}$ to $\mathrm{S}_{4}$; the oxidation of water and release of oxygen then occur rapidly in the dark, returning the complex to $\mathrm{S}_{0}$. Recent medium-resolution X-ray diffraction analyses of crystals from prokaryotic PSII (thermophilic cyanobacteria) have shown the location of the $\mathrm{Mn}-\mathrm{Ca}$ complex and pigments within the protein framework $(3-5)$.

It is well-established that in eukaryotic PSII (plants), the Mn complex contains $\mu$-oxo-bridged pairs and that pairs in two different orientations with respect to the membrane plane can be characterized by EXAFS (6-8). Both X-ray and EPR spectroscopy can detect changes in the mean oxidation state of the Mn, clearly indicating that the complex is the oxidant 
accumulator for water oxidation. The $\mathrm{S}_{0}(9,10)$ and $\mathrm{S}_{2}(11)$ intermediates have electron net $1 / 2$ spin states with $\mathrm{Mn}$ hyperfine structured (multiline) EPR spectra that can be detected by standard perpendicular mode EPR. The $S_{1}$ and $\mathrm{S}_{3}$ states are normally considered to be EPR silent, or unresolved under these conditions, but have been shown to have integer spin signals that can be detected by parallel mode EPR (12-14).

The mechanism of water oxidation is still unknown. Although many models have been suggested, the site of water binding and oxidation has not been unequivocally shown. Identification of the Mn complex as the oxidant accumulator has provided support for the proposal that it is also the catalytic site. However, it has only recently been directly demonstrated that substrate water actually binds to $\mathrm{Mn}$ in at least one intermediate in the catalytic cycle $\left(S_{2}\right)(15)$. This involved detection of ${ }^{17} \mathrm{O}$ ESEEM modulations on the $\mathrm{S}_{2}$ multiline signal, following partial exchange of solvent water with ${ }^{17} \mathrm{O}$-labeled water. The hyperfine characteristics of the interaction suggested direct ligation of water to Mn.

An alternative strategy for detecting interaction of water with the paramagnetic Mn center in PSII is to measure the ${ }^{1} \mathrm{H}$ or ${ }^{2} \mathrm{H}$ hyperfine couplings arising from natural abundance or $\mathrm{D}_{2} \mathrm{O}$-substituted solvent water. This has numerous practical advantages over the use of $\mathrm{H}_{2}{ }^{17} \mathrm{O}$, which is expensive and generally available only at moderate enrichment $(\sim 50 \%)$. Moreover, detailed interpretation of the ${ }^{17} \mathrm{O}$ ESEEM is difficult, as the ${ }^{17} \mathrm{O}$ nuclear spin is $5 / 2$ and the magnetic interaction with the Mn involves significant quadrupolar as well as anisotropic hyperfine effects (16). Interactions of ${ }^{1} \mathrm{H}$ or ${ }^{2} \mathrm{H}$ from bound water are relatively simple to analyze, in either ESEEM or ENDOR, as quadrupolar effects are either absent or small and the coupling is generally dominated by dipolar interaction between the H/D nucleus and the effective electron spin density on the metal ion binding the water. This is often true even in metal clusters, due to the inverse cube distance dependence of the dipolar interaction. Typically then, only close nuclear-electron spin effects contribute significantly to the total interaction. The difficulty in using ${ }^{1} \mathrm{H}$ or ${ }^{2} \mathrm{H}$ hyperfine sensitive spectroscopies is distinguishing those proton/deuteron couplings due to interaction of water with the paramagnetic center from interactions of hydrogen or an exchangeable deuteron with other nearby groups, particularly in a protein matrix. Nonetheless, techniques such as ${ }^{2} \mathrm{H}-{ }^{1} \mathrm{H}$ ESEEM or ${ }^{1} \mathrm{H}$ ENDOR are so experimentally convenient and sensitive that a number of studies have employed these methods to assess interactions of a proton (17-20), exchangeable deuteron $(21,22)$, and small molecule $(23-27)$ with the Mn cluster in PSII.

Kinetic isotope exchange studies by Wydrzynski et al. (28, 29) using $\mathrm{H}_{2}{ }^{18} \mathrm{O}$ indicate that water is bound to the water oxidation complex in $\mathrm{S}_{0}$ and remains exchangeable until $\mathrm{S}_{3}$. They generally detect two rates of exchange in each $\mathrm{S}$ state, indicating two sites of water binding, one with significantly tighter binding than the other. As noted above, both the $S_{0}$ and $S_{2}$ states exhibit well-characterized $1 / 2$ net spin EPR multiline signals, suitable for ESEEM or ENDOR study, using $\mathrm{CW}$ or pulsed techniques. To date, virtually all such studies for detecting interaction of small molecules with the electron spin giving rise to the multiline signal have been performed in the $S_{2}$ state, generated by continuous lowtemperature $(\sim 200 \mathrm{~K})$ illumination of suitably prepared samples frozen in $\mathrm{S}_{1}$. This technique is widely used in EPR spectroscopy with PSII and is very convenient. It permits the use of concentrated samples but does not lend itself to generation of the $\mathrm{S}_{0}$ state, which normally requires flash advancement from $\mathrm{S}_{1}$ (three single-turnover flashes, $T>0$ ${ }^{\circ} \mathrm{C}$ ), followed by freeze trapping. Sample chlorophyll concentrations must then be sufficiently low (typically $<3 \mathrm{mg}$ / $\mathrm{mL}$ ) to allow efficient flash illumination and coherent $\mathrm{S}$ state advancement. Recently, Britt et al. (22) have reported ${ }^{2} \mathrm{H}$ ESEEM from the $\mathrm{S}_{0}$ state of PSII, using a flash turnoverchemical trapping technique with $\mathrm{D}_{2} \mathrm{O}$-exchanged membrane particles (30). These data implied a greater number of exchangeable deuterons interacting with the Mn cluster in the $S_{0}$ state, compared to the $S_{2}$ state, with stronger effective hyperfine interactions (i.e., closer).

Ahrling et al. first showed that a Mn hyperfine structured signal from the $S_{0}$ state could be generated by multiple-flash turnover of PSII membrane particles (10). Apart from the presence of $\mathrm{MeOH}$ at $\sim 1 \mathrm{M}$ in the solvent buffer, the conditions used were essentially "physiological", with the $\mathrm{S}_{0}$ signal being still detectable after two full turnover cycles of the enzyme (seven flashes). At concentrations up to several molar, $\mathrm{MeOH}$ does not inhibit the water splitting function (25), and its presence is normally believed to be necessary to render visible the hyperfine structure of the $S_{0}$ state, whether generated by chemical reduction (9) or flash advancement $(10,30)$. The basis of this effect is still unclear; the $\mathrm{S}_{2}$ multiline signal seen in the presence of $\mathrm{MeOH}$ is subtly different from that seen in its absence and appears to be composed of two components under some conditions (24, 27). Formation of the $g=4.1$ signal, the other nonintegral spin state seen in $S_{2}$, is efficiently suppressed by $1 \mathrm{M} \mathrm{MeOH}$ (31). The fact that $\mathrm{MeOH}$, uniquely among small alcohols, has these properties means that its interaction with the $\mathrm{Mn}$ cluster in PSII has long been a subject of interest.

Until recently, ESEEM investigations of PSII have been performed on concentrated samples, unsuitable for flash turnover. We have taken advantage of improved signal-tonoise ratios in new commercial instrumentation to investigate the interaction of exchangeable protons, using deuterated water $\left(\mathrm{D}_{2} \mathrm{O}\right)$, and of methanol, using methyl $d_{3}$-labeled $\mathrm{MeOH}(\mathrm{DMeOH})$. These studies probe the $\mathrm{S}_{0}$ and $\mathrm{S}_{2}$ states produced by laser flash turnover of dark-adapted $\left(S_{1}\right)$ samples of spinach PSII preparations with chlorophyll concentrations of $2-3 \mathrm{mg} / \mathrm{mL}$. The results are compared to those obtained with $\mathrm{S}_{2}$ samples of pea and spinach PSII prepared in the more conventional way by $200 \mathrm{~K}$ illumination of concentrated $(10 \mathrm{mg} / \mathrm{mL}$ chlorophyll) samples. Fitting of the time domain data provides information about the likely binding of water and methanol to the $\mathrm{Mn}-\mathrm{Ca}$ cluster.

\section{MATERIALS AND METHODS}

\section{Sample Preparation}

PSII membrane fragments were made from spinach (10) or from 10-14-day-old pea seedlings, as described by Ford and Evans (32). Control rates of oxygen evolution for PSII membranes were 500-1100 $\mu \mathrm{mol}$ of $\mathrm{O}_{2}(\mathrm{mg} \text { of chl })^{-1} \mathrm{~h}^{-1}$ using ferricyanide and dimethylbenzoquinone as electron acceptors and measured in an oxygen electrode at $298 \mathrm{~K}$. The membranes were stored at $77 \mathrm{~K}$ in $20 \mathrm{mM}$ MES, 15 $\mathrm{mM} \mathrm{NaCl}, 5 \mathrm{mM} \mathrm{MgCl}_{2}$, and $0.4 \mathrm{M}$ sucrose (pH 6.3). The 
chlorophyll concentration was measured by the method of Porra (33). Membrane samples, after thawing on ice, were washed twice in a buffer $(20 \mathrm{mM}$ MES, $10 \mathrm{mM} \mathrm{NaCl}, 5$ $\mathrm{mM} \mathrm{CaCl} 2,10 \mathrm{mM} \mathrm{MgCl}_{2}$, and $400 \mathrm{mM}$ sucrose) made up in $\mathrm{D}_{2} \mathrm{O}$ or $\mathrm{H}_{2} \mathrm{O}$ and adjusted to a proton equivalent $\mathrm{pH}$ of 6.0. The samples were made up to $\sim 1.4-1.9 \mathrm{mg}$ of $\mathrm{chl} / \mathrm{mL}$ in the same buffer with $1 \mathrm{mM}$ EDTA and $0.5 \mathrm{mM}$ PpBQ with or without alcohol additions $(\mathrm{MeOH}, \mathrm{DMeOH}$, or EtOH at $\sim 1 \mathrm{M}$ ). Flash illumination was performed using a $\mathrm{Nd}$ : YAG laser (350 mJ per $7 \mathrm{~ns}$ flash at $532 \mathrm{~nm}$ ) as described previously (10). This involves a preflash procedure that synchronizes all centers in $\mathrm{S}_{1}$ prior to flash advancement. Samples for $200 \mathrm{~K}$ illumination were prepared at $10 \mathrm{mg}$ of $\mathrm{chl} / \mathrm{mL}$ in the same manner described above, given a preillumination light treatment at room temperature, with dark adaptation for $3 \mathrm{~h}$ on ice, and then illuminated with continuous white light at $200 \mathrm{~K}$ in an ethanol-dry ice bath for $10 \min (24)$.

\section{CW EPR}

CW EPR was performed on a Bruker ESP300E spectrometer equipped with an Oxford Instruments cryostat. Spectrometer conditions were as follows: frequency, $9.43 \mathrm{GHz}$; modulation amplitude, $20 \mathrm{G}$. For $\mathrm{S}_{0}$, the temperature was 5 $\mathrm{K}$ and the microwave power $15.5 \mathrm{~mW}$. For $\mathrm{S}_{2}$, the temperature was $7.5 \mathrm{~K}$ and the microwave power $6.32 \mathrm{~mW}$.

\section{ESEEM}

Experimental. ESEEM spectra were recorded at $4 \mathrm{~K}$ on a Bruker ESP580-U389R ELEXSYS X-band pulsed spectrometer equipped with a Bruker EN 4118 X-MD-4W flexline resonator and an Oxford Instruments CF395 cryostat. ESEEM spectra were recorded using a standard three-pulse ESEEM program with phase cycling (Bruker X-EPR software). The pulse sequence was $\pi / 2-\tau-\pi / 2-T-\pi / 2-$ echo, where $\pi / 2=16 \mathrm{~ns}, \tau=128 \mathrm{~ns}$, and $\mathrm{T}$ varies from 32 to $8000 \mathrm{~ns}$ in $8 \mathrm{~ns}$ steps. The decay of the echo over a longer time period was measured using a $52 \mathrm{~ns}$ step time. The value of $\tau$ is set at $128 \mathrm{~ns}$ to suppress the free proton modulation. This results in some attenuation of weakly coupled deuterium modulations (to $\sim 65 \%$ of maximum values) but means loss of only the first $\sim 150 \mathrm{~ns}$ of echo response. The spectra have 1024 points and were recorded with 100 shots/point for the $2 \mathrm{mg} / \mathrm{mL}$ flash samples and 60 shots/point for the $10 \mathrm{mg} /$ $\mathrm{mL} 200 \mathrm{~K}$ illuminated samples. The final spectra are the sum of four spectra accumulated during phase cycling. All spectra were acquired at a field position of $3550 \mathrm{G}$, a spectrometer frequency of $9.71 \mathrm{GHz}$, and a temperature of $4 \mathrm{~K}$. Samples prepared by flash illumination were shipped from Canberra to London in a liquid nitrogen transport dewar. ESEEM spectra were recorded 5-7 days after sample preparation. Analysis of control samples indicated that there was little or no decay of the EPR signals over this period. Samples for $200 \mathrm{~K}$ illumination were prepared in London as described above.

Data Analysis. ${ }^{2} \mathrm{H}$ ESEEM echo decay modulation spectra were modeled using the exact expressions for $1 / 2$ spin electron and 1 spin nuclei (ref 34 and Appendix 2), assuming zero nuclear quadrupole interaction. Detailed simulations (Chapter 7 of ref 34) of the X-band deuterium ESEEM response show that when multiple interactions strongly attenuate the modu-

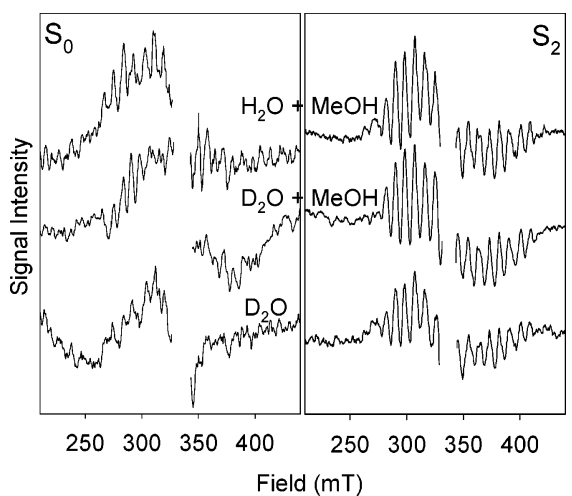

FIGURE 1: CW multiline spectra for PSII samples predominantly in the $\mathrm{S}_{0}$ state (three-flash) and $\mathrm{S}_{2}$ state (one-flash), as indicated. See Materials and Methods for spectrometer conditions. Solvent buffer conditions are indicated in each case. PSII concentrations were $\sim 2 \mathrm{mg}$ of $\mathrm{chl} / \mathrm{mL}$. EPR conditions are given in Materials and Methods. All spectra have the $S_{1}$ (zero-flash) background subtracted. The radical region around $g=2$ has been removed.

lations beyond $\sim 2 \mu \mathrm{s}$, as is the case here, ignoring the quadrupole terms produces barely detectable effects for typical interaction geometries. Up to four classes of separate deuteron interactions were included, with variable numbers of equivalent nuclei within each class. Point dipole nuclearelectron spin and isotropic hyperfine interactions were included for each class. Explicit numerical powder pattern averages of the $V_{\alpha}(\tau T)$ and $V_{\beta}(\tau T)$ angular-dependent modulation branch functions were computed for each deuteron class and combined according to the exact three-pulse product expression (Appendix 2). No attempt was made to correlate the angular dependences of the individual deuteron interactions, either within or between classes, according to some assumed structural model. An explicit form for the echo amplitude decay function was assumed, on the basis of the observed shape of this function over a long time and its qualitative similarity to the $T_{1}$ decay behavior seen earlier in relaxation studies of tyrosine D in PSII (ref 35 and Appendix 1).

\section{RESULTS}

\section{CW EPR Spectra}

Figure 1 shows representative $\mathrm{CW}$ light minus $\mathrm{S}_{1}$ dark multiline spectra for flash turnover samples, in the $S_{2}$ (one flash) and $\mathrm{S}_{0}$ (three flashes) states, as obtained here. The flash turnover efficiency in these experiments is such that no more than $10 \%$ of centers are in the $S_{2}$ state after three flashes (see ref 27 and below). The $\mathrm{S}_{2}$ spectra in the presence of $\mathrm{D}_{2} \mathrm{O}$ are both very similar to the $\mathrm{H}_{2} \mathrm{O}+\mathrm{MeOH}$ spectrum, irrespective of whether $\mathrm{MeOH}$ is present with the $\mathrm{D}_{2} \mathrm{O}$. It is interesting to see that a hyperfine structured $\mathrm{S}_{0}$ signal is observable in the $\mathrm{D}_{2} \mathrm{O}$ sample lacking $\mathrm{MeOH}$, something that does not occur with $\mathrm{H}_{2} \mathrm{O}$ medium. This non- $\mathrm{MeOH} \mathrm{S}_{0}$ signal is prominent downfield and differs significantly in shape from the $\mathrm{MeOH} \mathrm{S} \mathrm{S}_{0}$ spectra seen in $\mathrm{H}_{2} \mathrm{O}$ or $\mathrm{D}_{2} \mathrm{O}$ solvent, the latter two being quite similar.

\section{ESEEM}

$\mathrm{DMeOH}$ Binding. Figure 2A shows the three-pulse ESEEM time domain results for a flash turnover series on spinach PSII membranes, in the presence of $1 \mathrm{M} \mathrm{DMeOH}$ 


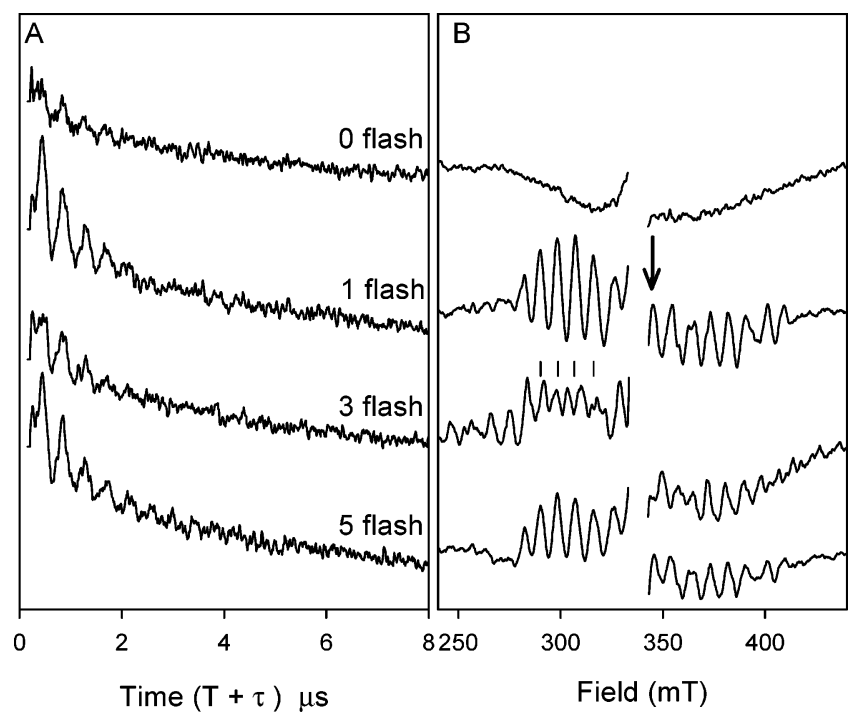

FIGURE 2: (A) Three-pulse time domain ESEEM for a flash turnover series in $\mathrm{H}_{2} \mathrm{O} / \mathrm{DMeOH}$ buffer, as described in Materials and Methods (acquisition duration of $8 \mu \mathrm{s}$ ). These are spectra as acquired, with digital filtering. Traces are arbitrarily offset along the signal axis. The deuterium modulation seen on zero-flash spectrum is somewhat variable, and that shown here is typical. (B) Corresponding CW spectra (conditions as in Figure 1) for flash series. These have a buffer only background subtracted. The arrow in panel B shows the position in the multiline spectrum equivalent to the field position at which the ESEEM spectra were acquired. Markers above the three-flash spectrum at low field indicate positions of the four most intense peaks of the $S_{2}$ multiline signal (see the text).

$\left(\mathrm{H}_{2} \mathrm{O} / \mathrm{DMeOH}\right.$ medium). Figure 2B shows the corresponding $\mathrm{CW}$ spectra for this series. The equivalent field position of the ESEEM acquisition point in the multiline pattern is shown in Figure 2B (arrow). There is weak deuterium (and probably ${ }^{14} \mathrm{~N}$ ) modulation on the zero-flash echo envelope. This was somewhat variable between samples and could be undetectable in some cases. The origin of this variability is unknown. Unlike the case in some studies on PSII membranes, the $\mathrm{S}_{1}$ state sample "background" here is relatively featureless in a wide range around $g=2$ (Figure $2 \mathrm{~B}$ ). There are no detectable Kramers state signals $\left(\mathrm{Cu}^{2+}, \mathrm{Q}_{\mathrm{A}}{ }^{-}\right.$, ferricytochrome, etc. $)$ outside the central radical region, but a very broad, roughly Gaussian $g \sim 2$ signal appears to be present (see the Supporting Information). Figure 2 shows that the characteristic one-flash $S_{2}$ pattern substantially returns after five flashes, with $\sim 70 \%$ efficiency, by either ESEEM modulation or CW multiline amplitude. This is consistent with the previously shown turnover behavior of these samples. Kok analysis (27) indicates that the one-flash signal is $>90 \%$ pure $\mathrm{S}_{2}$ and the three-flash signal is $\sim 75 \%$ pure $\mathrm{S}_{0} .{ }^{2}$ The level of residual $\mathrm{S}_{2}$ in the three-flash sample is $<10 \%$, as is also evident from the alignment of the four most intense $S_{2}$ multiline peak positions in the three-flash spectrum (Figure 2B).

Quantitative interpretation of the ESEEM patterns requires that the form of the intrinsic echo amplitude attenuation

\footnotetext{
${ }^{2}$ In fact the situation is, fortuitously, somewhat better than this. Because the three $\mathrm{DMeOH}$ deuteron couplings are very similar in $\mathrm{S}_{2}$ and $\mathrm{S}_{0}$ (see Table 2), the residual $\sim 10 \%$ of $\mathrm{S}_{2}$ in the three-flash spectra means that the latter ESEEM is indistinguishable, within our signalto-noise ratio, to an $\sim 90 \%$ "pure" $\mathrm{S}_{0}$ response. The data are not adequate to attempt deconvolutions beyond this level.
}

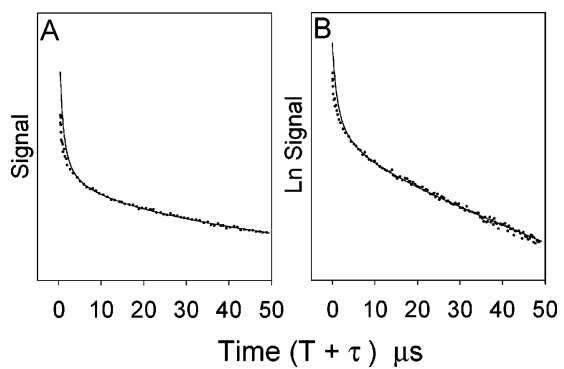

FIGURE 3: Echo decay plots [linear (A) and ln amplitude (B)], over an extended acquisition duration $(50 \mu \mathrm{s})$, for a typical $\mathrm{H}_{2} \mathrm{O} / \mathrm{DMeOH}$ sample in the $S_{2}$ multiline state (points). Shown also (solid lines) are the fitted curves for the intrinsic echo decay function using the dipolar relaxation enhancement model described in the text. This passes through the peaks of the data modulations at short times, barely discernible in the figure. The relaxation model parameter values are as follows: $k_{\mathrm{d}}=1.3 \times 10^{6} \mathrm{~s}^{-1}, k_{\text {background }}=1.2 \times 10^{4}$ $\mathrm{s}^{-1}$, and $F=0.63$.

function be known, or accounted for. This is in part because the initial signal decay, which establishes the true echo amplitude at zero time, is lost in the three-pulse sequence, even without probe dead time effects. Usually, some totally empirical function is fitted (exponential polynomial in time etc.; e.g., see ref 36). Because of the nature of the threepulse sequence, a lower bound for the intrinsic echo decay rate is the electron spin lattice $\left(T_{1}\right)$ relaxation rate, but the echo decay is usually faster due to cross relaxation effects and may be highly nonexponential (37). Alternatively, the modulation envelope containing the interaction of interest (e.g., from a ${ }^{2} \mathrm{H}$-labeled species) can be ratioed with an equivalently generated envelope obtained with the nonlabeled (e.g., ${ }^{1} \mathrm{H}$ ) species. While under favorable circumstances this accounts for the long time signal attenuation, it does not correct for the initial signal loss. In addition, it inevitably reduces the signal-to-noise ratio in the isolated modulation pattern from the label, which is a major consideration in these experiments where dilute samples must be employed for coherent $\mathrm{S}$ state advancement. For these and other reasons, it was considered preferable to determine the explicit shape of the decay function and use this in the simulation fits to the time domain ESEEM data for deuterated $\mathrm{MeOH}$ and water interactions.

Figure 3 shows the echo decay (linear and $\ln$ plots), over an extended duration $(50 \mu \mathrm{s})$, for a typical $\mathrm{H}_{2} \mathrm{O} / \mathrm{DMeOH}$ sample in the $\mathrm{S}_{2}$ multiline state. At times of $>5 \mu \mathrm{s}$, the decay is a simple exponential for a substantial fraction of the sample, but at shorter times, it is highly nonexponential. The behavior is, however, qualitatively very similar to that recently seen by Mamedov et al. for the spin lattice relaxation of the redox active tyrosine $\mathrm{Y}_{\mathrm{D}}{ }^{\bullet}$ in PSII core complexes (35) ( $S_{1}$ or $S_{2}$ state), and the same phenomenological model was used to fit the data here (Appendix 1). In this work, the intrinsic (background) relaxation rate of the paramagnet is slow and exponential, but dipolar relaxation interaction with a distant fast relaxing species occurs in a variable fraction $(F)$ of centers. Figure 3 shows that the model fits the threepulse echo decay very well. Comparable results are seen for the $S_{1}$ and $S_{0}$ echo decays, except that the background (but not dipolar) rates are typically different from those seen in $\mathrm{S}_{2}$.

Figure 4 shows sample-averaged results for the time domain (A and B) and frequency domain $(\mathrm{C}$, Fourier cosine 

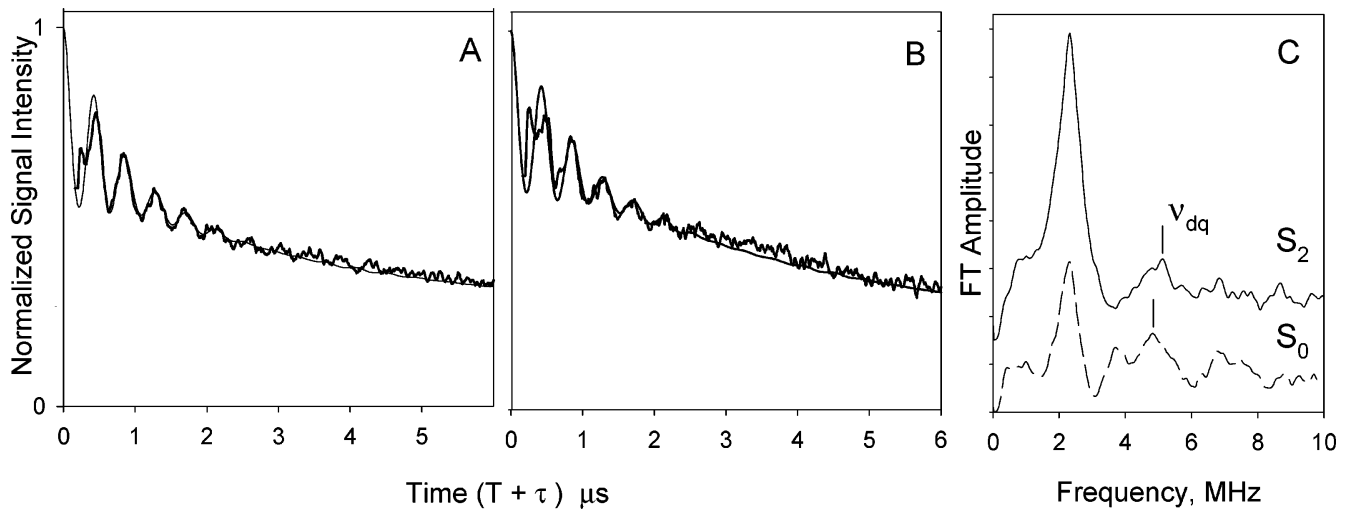

FIGURE 4: Sample-averaged (two series) three-pulse time domain ESEEM for $\mathrm{H}_{2} \mathrm{O} / \mathrm{DMeOH}$ samples from flash turnover series (Materials and Methods, $8 \mu \mathrm{s}$ acquisition). Data are normalized to an initial amplitude of 1 at zero time (using echo decay fits, as in Figure 3): (A) one flash $\left(\mathrm{S}_{2}\right)$ and $(\mathrm{B})$ three flashes (mainly $\mathrm{S}_{0}$ ). Smooth lines through data in panels A and B are calculated ESEEM fits (see the text) using the three-methyl deuteron coupling parameter set from Table 1. Echo decay $F$ values are 0.78 in both cases. Slight deviations of the echo decay response from a smooth function in the region beyond $\sim 3 \mu$ s cannot be reproduced and are presumed to be machine artifacts. Shown in panel $\mathrm{C}$ are the cosine Fourier transforms of the echo modulation data from panels A and B (the $\mathrm{S}_{2}$ spectrum is vertically offset). The prominent peak at $\sim 2.3 \mathrm{MHz}$ is from the deuteron coupling. Markers show peaks corresponding to ${ }^{14} \mathrm{~N}$ double-quantum frequencies $\left(v_{\mathrm{dq}}\right)$ at $4.8\left(\mathrm{~S}_{0}\right)$ and $5.1 \mathrm{MHz}\left(\mathrm{S}_{2}\right)$ (see the text). Low-frequency $\alpha$ branch transitions $(<2 \mathrm{MHz})$ are more evident in the $\mathrm{S}_{0}$ frequency domain spectrum.

\begin{tabular}{|c|c|c|c|c|c|c|c|}
\hline \multirow[b]{2}{*}{ deuteron $^{a}(i)$} & \multirow[b]{2}{*}{$T_{\mathrm{d} i}(\mathrm{MHz})$} & \multirow[b]{2}{*}{$B_{i}^{b}$} & \multirow[b]{2}{*}{$A_{i}(\mathrm{MHz})$} & \multicolumn{2}{|c|}{$|\rho|=2^{e}$} & \multicolumn{2}{|c|}{$|\rho|=1^{e}$} \\
\hline & & & & $\overline{r_{i}^{c}(\AA)}$ & $\mathrm{Mn}-\mathrm{O}^{d}(\AA)$ & $\overline{r_{i}^{c}(\AA)}$ & $\mathrm{Mn}-\mathrm{O}^{d}(\AA)$ \\
\hline & & & $\mathrm{S}_{2}$ & & & & \\
\hline 1 & $0.64 \pm 0.03$ & $0.28 \pm 0.01$ & $0.18 \pm 0.03$ & 3.34 & & 2.65 & \\
\hline 2 & $0.39 \pm 0.03$ & $0.17 \pm 0.01$ & $\sim 0.0$ & 3.95 & & 3.13 & \\
\hline \multirow[t]{2}{*}{3} & $0.39 \pm 0.03$ & $0.17 \pm 0.01$ & $\sim 0.0$ & 3.95 & & 3.13 & \\
\hline & & & & & 1.92 & & $\sim 1.2$ \\
\hline & & & $\mathrm{S}_{0}$ & & & & \\
\hline 1 & $0.60 \pm 0.03$ & $0.26 \pm 0.01$ & $0.14 \pm 0.03$ & 3.43 & & 2.72 & \\
\hline 2 & $0.37 \pm 0.03$ & $0.16 \pm 0.01$ & $\sim 0.0$ & 4.03 & & 3.20 & \\
\hline \multirow[t]{2}{*}{3} & $0.37 \pm 0.03$ & $0.16 \pm 0.01$ & $\sim 0.0$ & 4.03 & & 3.20 & \\
\hline & & & & & 1.96 & & $\sim 1.3$ \\
\hline
\end{tabular}

\footnotetext{
${ }^{a}$ Assumes only one $\mathrm{MeOH}$ molecule interacting with the cluster. See the text. ${ }^{b}$ Corresponds to an $H_{0}$ of 3550 G. For dipolar coupling, $T_{\mathrm{d}}=B v_{\mathrm{I}}$ $\mathrm{MHz}$, where $\nu_{\mathrm{I}}$ is the nuclear Zeeman frequency. See the text. ${ }^{c}$ Calculated from the point dipole model assuming the given $\rho$ value. See the text. ${ }^{d}$ Calculated from the model geometry in Figure 9A. ${ }^{e}$ Typically expected limits for $\rho$ magnitude values. See the text.
}

transform) ESEEM from PSII membranes in $\mathrm{H}_{2} \mathrm{O} / \mathrm{DMeOH}$ medium, for the $\mathrm{S}_{2}$ and $\mathrm{S}_{0}$ multiline states. Indicated also are best fit simulations of the three-pulse echo data, using the procedure described in Materials and Methods and the parameter values listed in the figure caption of Figure 4 and Table 1. It was found that three single-deuteron interactions gave a good fit to the modulation pattern, corresponding to a single $\mathrm{DMeOH}$ molecule binding close to the multiline Mn cluster in both $S_{2}$ and $S_{0}$. The fitting yields two hyperfine interaction parameters for each deuteron, the dipolar interaction term $T_{\mathrm{d}}$ and the isotropic contact term $A$ (see Analysis and Discussion and Appendix 2). In these simulations, no "zero-flash background" was subtracted, a matter we return to in Analysis and Discussion.

At short times, high-frequency $(4-5 \mathrm{MHz})$ modulations are apparent on the echo responses in panels $\mathrm{A}$ and $\mathrm{B}$ of Figure 4 (see also Figure 8 below). In the $S_{2}$ state, these are known to arise from double-quantum ${ }^{14} \mathrm{~N}$ couplings, due to a single histidine ligand to the Mn cluster (D1-His332) (38, 39). Figure $4 \mathrm{~B}$ is the first demonstration that such modulations are also seen in $\mathrm{S}_{0}$. The frequency transforms in Figure 4C show also components below $2 \mathrm{MHz}$, which are likewise characteristic of ${ }^{14} \mathrm{~N}$ interactions and arise from the $\alpha$ branch single-quantum transitions (34).
$\mathrm{D}_{2} \mathrm{O}$ Binding Exchange. Figure 5 shows representative results for the time domain ESEEM from PSII membranes in $\mathrm{D}_{2} \mathrm{O} / \mathrm{MeOH}$ (A and $\mathrm{B}$ ) and $\mathrm{D}_{2} \mathrm{O}(\mathrm{C}$ and $\mathrm{D})$ media, for the flash-generated $\mathrm{S}_{2}$ and $\mathrm{S}_{0}$ multiline states. Indicated also are best fit simulations of the three-pulse echo data (Materials and Methods) using the parameter values listed in the figure captions and Table 2. In all cases, adequate fits could be obtained assuming the same basic four-deuteron shell model, with variable numbers $\left(n_{i}\right)$ of equivalent deuterons in each shell (labeled, $i=1-4$ ). Shells 1 and 2 correspond to the most strongly interacting deuterons, and the occupancy of these shells did not vary with $\mathrm{S}$ state or $\mathrm{MeOH}$ addition. Shell 4, containing the most weakly interacting deuterons, was similarly constant within the resolution of the data. Shell 3 , however, was highly variable, with $n_{3}$ ranging from 0 to $\sim 10$ (see below). For flash turnover samples, $n_{3}$ was generally between 5 and 9 . The hyperfine parameters (particularly the $T_{\mathrm{d} i}$, to which the fitting is most sensitive) varied little with $\mathrm{S}$ state or $\mathrm{MeOH}$ addition, however. This was also the case if the larger alcohol, EtOH, was added in place of $\mathrm{MeOH}$ (not shown).

Results of Turnover at $200 \mathrm{~K}$. Prior to this work, all ESEEM studies on the $S_{2}$ state involved generation of the $1 / 2$ spin multiline by continuous low-temperature $(\sim 200 \mathrm{~K})$ 


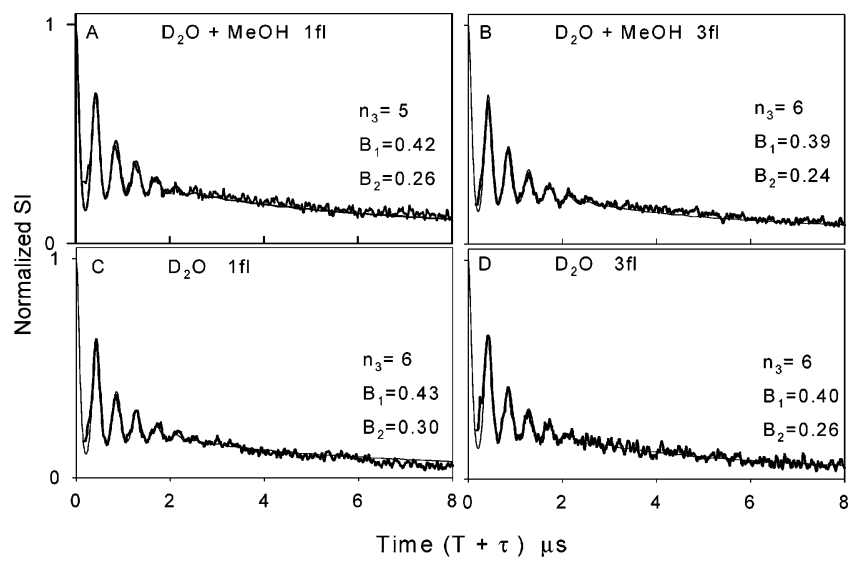

FIGURE 5: Normalized (as in Figure 4) three-pulse time domain ESEEM for $\mathrm{D}_{2} \mathrm{O} / \mathrm{MeOH}(\mathrm{A}$ and $\mathrm{B})$ and $\mathrm{D}_{2} \mathrm{O}(\mathrm{C}$ and $\mathrm{D})$ samples from flash turnover series (Materials and Methods, $8 \mu$ s acquisition) for $\mathrm{S}_{2}(\mathrm{~A}$ and $\mathrm{C})$ and $\mathrm{S}_{0}(\mathrm{~B}$ and $\mathrm{D})$. Smooth lines through data are calculated ESEEM fits using the four-shell deuteron coupling model (see the text and Table 2). Individual sample parameter values for shells $1-3$, relevant to the discussion in text, are given in each panel. Differences between the data and fits at earliest times are due to the presence of ${ }^{14} \mathrm{~N}$ modulations (as in Figure 4; see also Figure 8).

Table 2: Average ESEEM Simulation Parameters for $\mathrm{D}_{2} \mathrm{O}$ Interaction in the $S_{2}$ and $S_{0}$ States

\begin{tabular}{cccccc}
\hline $\begin{array}{c}\text { deuteron } \\
(i)\end{array}$ & $\begin{array}{c}\text { number } \\
\left(n_{i}\right)\end{array}$ & $T_{\mathrm{di} i}{ }^{a, b}(\mathrm{MHz})$ & $B_{i}{ }^{a, b}$ & $A_{i}{ }^{a, b}(\mathrm{MHz})$ & $\begin{array}{c}{ }^{1} \mathrm{H} \text { ENDOR } \\
A_{\perp} \text { value }^{c} \\
(\mathrm{MHz})\end{array}$ \\
\hline & & & $\mathrm{S}_{2}{ }^{a}$ & & \\
$1^{d}$ & 1 & $0.97 \pm 0.05$ & $0.42 \pm 0.02$ & $0.32 \pm 0.03$ & 4.1 \\
$2^{d}$ & 1 & $0.65 \pm 0.03$ & $0.28 \pm 0.01$ & $0.27 \pm 0.03$ & 2.4 \\
3 & $\sim 0-10^{e}$ & $0.44 \pm 0.03$ & $0.19 \pm 0.01$ & $0.06 \pm 0.03$ & 2.4 \\
4 & 4 & $0.25 \pm 0.05$ & $0.11 \pm 0.02$ & $0.05 \pm 0.03$ & 1.3 \\
& & & $\mathrm{~S}_{0}{ }^{b}$ & & \\
$1^{d}$ & 1 & $0.90 \pm 0.05$ & $0.39 \pm 0.01$ & $0.27 \pm 0.02$ & \\
$2^{d}$ & 1 & $0.58 \pm 0.05$ & $0.25 \pm 0.01$ & $0.21 \pm 0.03$ & \\
3 & $\sim 5-8^{e}$ & $0.42 \pm 0.05$ & $0.18 \pm 0.01$ & $0.05 \pm 0.02$ & \\
4 & 4 & $0.25 \pm 0.05$ & $0.11 \pm 0.02$ & $0.05 \pm 0.02$ &
\end{tabular}

${ }^{a}$ Average of 10 results (except $n_{3}$ ) for flash, $200 \mathrm{~K}$ turnover, with or without $\mathrm{MeOH}$; spinach and pea PSII. See the text. Errors are approximately one standard deviation. Field $H_{0}=3550 \mathrm{G}$ for $\mathrm{S}_{2}$ and $\mathrm{S}_{0} \cdot{ }^{b}$ Average of five results (except $n_{3}$ ) for flash turnover, with or without $\mathrm{MeOH}$ and $\mathrm{EtOH}$; spinach PSII. ${ }^{c}$ Corresponding ${ }^{1} \mathrm{H}$ ENDOR coupling from ${ }^{2} \mathrm{H}$ hyperfine parameters, uncertainty $\pm 0.002 \mathrm{MHz}$ in all cases. See the text. ${ }^{d}$ Assumes only one $\mathrm{D}_{2} \mathrm{O}$ molecule strongly interacting with the cluster. See the text. ${ }^{e}$ Full range of $n_{3}$ for all samples that were examined. The fitting uncertainty for a given sample is $<2$. See the text.

illumination of concentrated PSII membrane samples, frozen in the dark $S_{1}$ state. It is useful to compare the results from this protocol with those obtained by room-temperature flash advancement. Figure 6 shows data for the time domain ESEEM of PSII membranes (10 mg of chl/ $/ \mathrm{mL}$ ) in $\mathrm{H}_{2} \mathrm{O} /$ $\mathrm{DMeOH}$ media, with the $\mathrm{S}_{2}$ multiline generated by $200 \mathrm{~K}$ illumination (Materials and Methods). Force et al. (25) have also assessed the interaction of $\mathrm{DMeOH}$ with the $\mathrm{S}_{2}$ multiline center by ESEEM. In these experiments, the $S_{2}$ state was generated by $200 \mathrm{~K}$ illumination of concentrated PSII particles, followed by a brief $4{ }^{\circ} \mathrm{C}$ annealing. These authors also found that a three-deuteron fit to their data gave an adequate simulation of the echo response, but the hyperfine interactions were approximately half the magnitude of those for the flash-generated $S_{2}$ state in Figure 4A (but with a

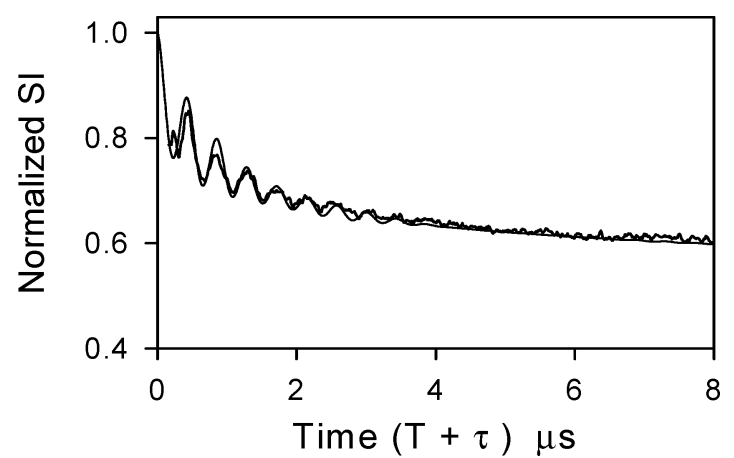

FIGURE 6: Normalized (as in Figure 4) three-pulse time domain ESEEM of PSII membranes (10 mg of chl $/ \mathrm{mL})$ in $\mathrm{H}_{2} \mathrm{O} / \mathrm{DMeOH}$ media, with the $S_{2}$ multiline signal generated by $200 \mathrm{~K}$ illumination (Materials and Methods, $8 \mu \mathrm{s}$ acquisition). The smooth line through the data is the calculated echo response, using the methyl deuteron dipolar coupling constants reported by Force et al. (25) $\left(T_{\mathrm{d}}=0.45\right.$, 0.20 , and $0.18 \mathrm{MHz}$; see also Figure 8 ). The $F$ value for the echo decay function is 0.38 . This is the lowest we have observed.

similar pattern of relative sizes). The simulation in Figure 6 uses the Force et al. parameters (see the figure caption). Although they are not optimized to our data, it is clear that these parameters reasonably reproduce the overall modulation depth, so our results are broadly similar to those reported earlier under such turnover conditions. However, compared to those generated with the flash protocol, PSII membrane samples prepared as in Figure 6 appear to be substantially heterogeneous. This is not obvious from the $\mathrm{CW}$ multiline spectra, which are superficially "normal" (e.g., see ref 24) but is in the $F$ value of the echo decay function. $F$ is generally 0.7-1.0 for the flashed samples but is less than 0.4 in Figure 6. Thus, a substantial fraction of the latter Mn centers see no "dipolar enhanced" relaxation. This would not of itself influence the modulation depth but may reflect some other structural variation at or near the site. We have previously shown that the multiline signal, as conventionally generated, is composed of two somewhat different forms, with very different implied interactions of bound $\mathrm{MeOH}$ (27). In fact, the data in Figure 6 are well-simulated, using our $\mathrm{DMeOH}$ coupling parameters, assuming that only approximately half of the centers exhibit resolved deuterium modulations (not shown; see Analysis and Discussion). The factors influencing this apparent heterogeneity are at present unclear. It is most pronounced in samples in $\mathrm{H}_{2} \mathrm{O}$ media with $200 \mathrm{~K}$ turnover (i.e., the typically employed experimental regime).

As mentioned earlier for the $\mathrm{D}_{2} \mathrm{O}$-exchanged samples, the population of moderately coupled deuterons in shell 3 was highly variable. This is dramatically illustrated in Figure 7. These are time domain ESEEM spectra from $200 \mathrm{~K}$ illumination $\mathrm{S}_{2}$ state samples, in $\mathrm{D}_{2} \mathrm{O} / \mathrm{MeOH}$ ( $\mathrm{A}$ and $\mathrm{C}$ ) and $\mathrm{D}_{2} \mathrm{O}$ (B and D) media. Panels $\mathrm{A}$ and $\mathrm{B}$ are for PSII membranes from spinach (as used in the flash series), and panels $\mathrm{C}$ and D are from pea PSII membranes (all $10 \mathrm{mg}$ of $\mathrm{chl} / \mathrm{mL}$ ). In all cases, the couplings for shells 1,2 , and 4 were very similar to those found in the flash experiments (populations identical), but there are major differences in the shell 3 populations (but not coupling). In pea PSII, shell 3 is virtually absent. It is from these data that the most reliable estimates of the shell 4 parameters are obtained (see Analysis and Discussion). In addition, $F$ is generally $>0.7$ for these $\mathrm{D}_{2} \mathrm{O}$-exchanged samples and may be systematically higher in pea PSII than in spinach PSII, for $200 \mathrm{~K}$ turnover. So 


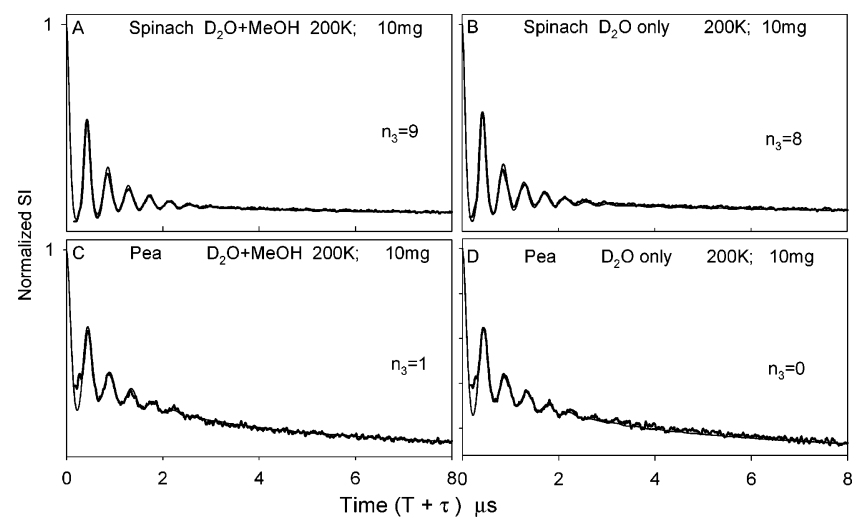

FIGURE 7: Normalized (as in Figure 4) three-pulse time domain ESEEM for $\mathrm{D}_{2} \mathrm{O} / \mathrm{MeOH}\left(\mathrm{A}\right.$ and $\mathrm{C}$ ) and $\mathrm{D}_{2} \mathrm{O}$ (B and $\mathrm{D}$ ) samples ( $10 \mathrm{mg}$ of $\mathrm{chl} / \mathrm{mL}$ ) in the $\mathrm{S}_{2}$ state generated by $200 \mathrm{~K}$ illumination (Materials and Methods, $8 \mu$ s acquisition). PSII membranes (10 $\mathrm{mg}$ of $\mathrm{chl} / \mathrm{mL}$ ) from spinach (A and B) and pea (C and D). Smooth lines through data are calculated ESEEM fits using the four-shell deuteron coupling model (see the text). In all cases, the parameter values are within the ranges given in Table 2; however, the highly variable shell 3 populations $\left(n_{3}\right)$ are given in each panel.

sample heterogeneity is a less important concern. For all the flash series data in $\mathrm{D}_{2} \mathrm{O}$-exchanged samples, $F$ was uniformly found to be 1.0 .

\section{ANALYSIS AND DISCUSSION}

The results show that there is strong ESEEM detectable on the $\mathrm{S}_{2}$ and $\mathrm{S}_{0}$ states by deuterons from both $d$-methanol and $\mathrm{D}_{2} \mathrm{O}$. The data for $\mathrm{S}_{2}$ generated by low-temperature turnover are generally consistent with earlier observations by ourselves and others $(21,22,24,25)$. Weaker modulation is also seen in the dark-adapted $S_{1}$ state. Conventionally, the $\mathrm{S}_{1}$ modulation has been considered to be background and subtracted or ratioed from the $200 \mathrm{~K}$ illuminated spectra to give the $S_{2}$ modulation spectrum. While this may be reasonable in samples with strong background absorption, e.g., from paramagnetic components such as $\mathrm{Cu}^{2+}$ in the light-harvesting proteins, our dilute spinach samples used in the flash experiments showed essentially no background signals in CW-EPR, except a very broad feature (Figure 2B; see also the Supporting Information). This feature is sensitive to turnover-induced $\mathrm{H}-\mathrm{D}$ exchange (Supporting Information), and we consider it reasonable that the signal arises from the $\mathrm{Mn}$ cluster in the $\mathrm{S}_{1}$ state. Then the weak modulation seen in the dark samples in fact arises from $S_{1}$. The latter would presumably be a weakly antiferromagnetically coupled even spin (probably $S=1$ ) excited state of the Mn cluster, which might be expected from the method of preparation (31). The zero-flash samples have no detectable Mn CW hyperfine structure and are poised in $\sim 100 \%$ $\mathrm{S}_{1}$ (Materials and Methods). Although $\mathrm{MeOH}$ suppresses the partially allowed, parallel polarization $\left(\Delta m_{\mathrm{s}}=0\right)$ signal associated with the paramagnetic $S_{1}$ state in intact PSII (31), nothing is known of its effect on the allowed, $\Delta m_{\mathrm{s}}= \pm 1$ transitions which would be active in ESEEM. These would occur in the $g \sim 2$ general region if the fine structure parameters inferred for the state in ref 31 obtained (i.e., a triplet system with D,E of $\sim 0.1 \mathrm{~cm}^{-1}$ or less). No attempt was made to analyze these $S_{1}$ modulations, as the relevant theory for systems with an electron and nuclear spin of $\geq 1$ is yet to be formulated. However, as a guide, in a triplet coupled to a $1 / 2$ nuclear spin, the ESEEM modulation depth is the same as that for the equivalent nuclear coupling to a $1 / 2$ electron spin, when the $\alpha$ and $\beta$ branch frequencies are close to $v_{\mathrm{I}}$, as here (see Appendix 2) (34). We have not therefore subtracted the "dark signal" in analyzing the data from two- and three-flash samples. It should be noted that failure to subtract a zero-flash decay in the ESEEM analysis of $\mathrm{S}_{2}$ or $\mathrm{S}_{0}$ is not responsible for the strong deuteron interactions we infer for $\mathrm{DMeOH}$ and $\mathrm{D}_{2} \mathrm{O}$ binding, below. While such subtraction slightly reduces the absolute amplitude of the deuteron oscillations, it results in a much greater reduction of the net echo decay amplitude. This means even stronger apparent coupling of the deuterons to the Mn spin center in $\mathrm{DMeOH}$ samples, to a degree that seems implausible in this case. For $\mathrm{D}_{2} \mathrm{O}$-exchanged samples, it leads to totally unphysical conclusions about the magnetization that can be attributed to the multiline states in $S_{2}$ or $S_{0}$ (see the Supporting Information). Due to the deep deuteron modulations seen in the flashed samples, no more than $\sim 15 \%$ of the zero-flash signal could be present without the unphysical requirement of a negative stimulated echo for the multiline signals. The maximum amount of $\mathrm{S}_{1}$ expected in the oneflash samples is $\sim 7 \%$, based on turnover yields (27). Our quantitative ESEEM analysis definitely rests on assuming little contribution from zero-flash components in the oneand three-flash spectra, but we could find no consistent interpretation of our data without making this assumption. The detailed reasoning is set out in the Supporting Information.

Fitting of the echo modulation requires knowledge of the intrinsic echo amplitude decay function (Figure 3). As noted above, this behavior is qualitatively very similar to that seen for the spin lattice relaxation of $\mathrm{Y}_{\mathrm{D}}{ }^{\bullet}$ in PSII core complexes (35) $\left(\mathrm{S}_{1}\right.$ or $\mathrm{S}_{2}$ state) or flash-advanced PSII membranes ( $\mathrm{F}$. Mamedov et al., unpublished observations). The redox active tyrosine radical $\mathrm{Y}_{\mathrm{D}}{ }^{\bullet}$ is located in the PSII reaction center near the same (luminal) membrane interface as the Mn cluster, but $\sim 30 \AA$ from the cluster (3). Its spin lattice relaxation in the temperature region of $4-30 \mathrm{~K}$ was described well by a model (ref 35 and Appendix 1) in which both slow intrinsic (background) relaxation and interaction enhanced dipolar relaxation with a distant fast-relaxing paramagnet occurred. This produced a much faster powder pattern averaged, nonexponential decay at short times but occurred only in a fraction, $F$, of the total centers. At $4 \mathrm{~K}, F$ was $\sim 0.6$ and approached 1 at higher temperatures. In fitting the multiline three-pulse echo decays, we find dipolar $\left(k_{\mathrm{d}}\right)$ and background ( $\left.k_{\text {background }}\right)$ rate parameters much faster than those seen for $\mathrm{Y}_{\mathrm{D}}{ }^{\bullet}$ but a similar value of $F$ at $4 \mathrm{~K}$. Comparable results are seen for the $S_{1}$ and $S_{0}$ echo decays, except that the background (but not dipolar) rates are typically different from those seen in $\mathrm{S}_{2}$. The $F$ and $k_{\mathrm{d}}$ parameters were found to vary somewhat in these membrane samples, for nonapparent reasons, which made ratioing signals problematic. The fact that the same basic relaxation model appears to hold for both $\mathrm{Y}_{\mathrm{D}}{ }^{\bullet}$ and the multiline Mn cluster is interesting and is currently under study. In the work presented here, the model has been used simply to empirically fit the echo decay behavior.

Data Fitting. To judge the reliability of the ESEEM simulation fits and the sensitivity of these to the various parameter values assumed within the multishell models, it 

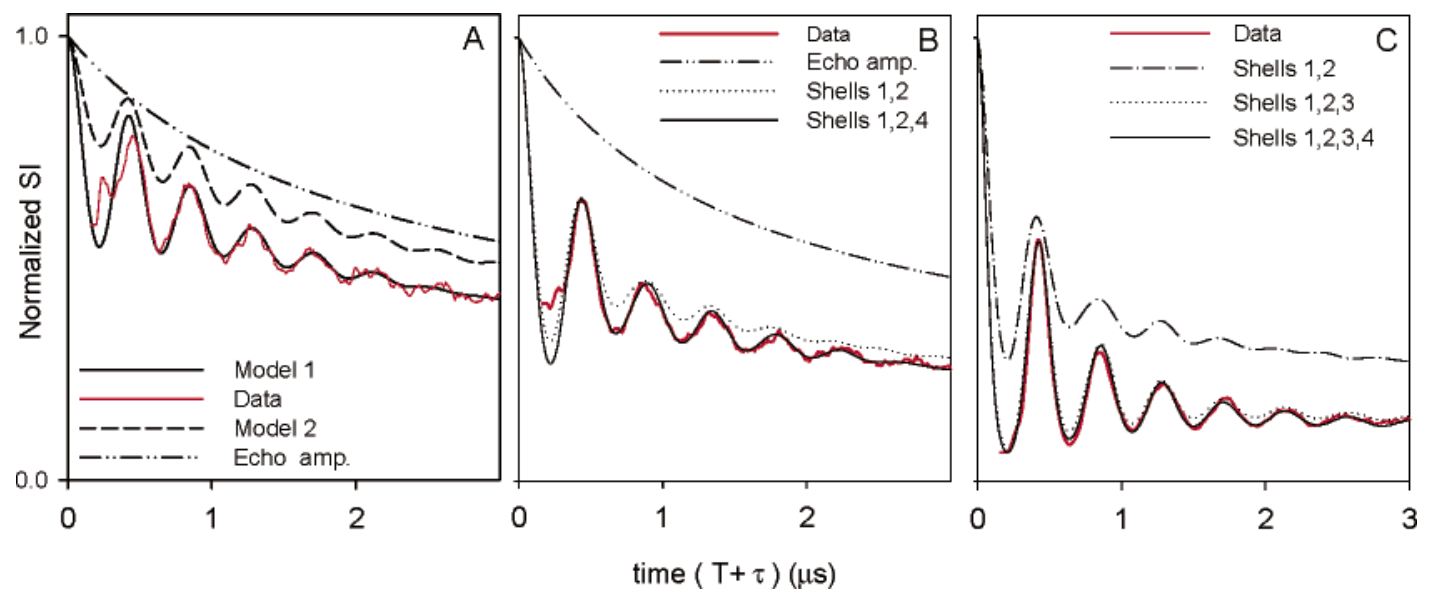

FIgURE 8: (A) Expanded version of Figure 4A at short time $(0-3 \mu \mathrm{s})$, for $\mathrm{D}_{3} \mathrm{MeOH}$ binding to the $\mathrm{S}_{2}$ multiline state in a flashed sample. The model 1 curve shows the full three-deuteron fit using parameters from Table 1. Indicated also (model 2) is the modeled ESEEM pattern corresponding to the parameter set from Force et al. (see Figure 6). The fitted intrinsic echo amplitude function alone is also shown (Echo amp.). Interference from ${ }^{14} \mathrm{~N}$ modulations with a frequency of $\sim 5 \mathrm{MHz}$ is evident below $0.5 \mu$ s. These damp quickly and produce negligible contributions beyond $\sim 0.7 \mu \mathrm{s}$. (B) Expanded version of Figure $7 \mathrm{D}$, for a $\mathrm{D}_{2} \mathrm{O}$-exchanged sample in $\mathrm{S}_{2}$ (zero shell 3 interactions). The model curves show progressively the fitted intrinsic echo amplitude function, the effects of the two strongly coupled deuterons (shells 1 and 2 ), and the full fit (shells 1,2, and 4), using parameter values within the ranges listed in Table 2 . The ${ }^{14} \mathrm{~N}$ modulation component is visible below $\sim 0.3 \mu \mathrm{s}$. (C) Expanded version of Figure 7A, for a $\mathrm{D}_{2} \mathrm{O}+\mathrm{MeOH}$-exchanged sample in $\mathrm{S}_{2}$ with maximal shell 3 interactions $\left(n_{3}=\right.$ 9). Progressive model interaction curves are also shown for shells 1 and 2 , shells $1-3$, and the full fit (shells $1-4$ ) using parameter values (other than $n_{3}$ ) within the ranges listed in Table 2. Refinement caused by inclusion of the shell 4 interactions is most evident at longer times, but the pattern is less sensitive to these than in panel B.

is useful to "decompose" the relevant contributions. The intrinsic echo decay function is necessary for correct normalization of the observed echo data, while the modulation pattern and depth are determined by the various hyperfine couplings, particularly the dipolar terms. Figure 8A shows an expanded version of Figure 4A at short time $(0-3 \mu \mathrm{s})$, for binding of $\mathrm{D}_{3} \mathrm{MeOH}$ to the $\mathrm{S}_{2}$ multiline state in a flashed sample. Apart from the obvious intrusion of highfrequency ${ }^{14} \mathrm{~N}$ components below $\sim 500 \mathrm{~ns}$, the simulation fit (model 1) is essentially within the experimental noise out to the limits of reliably detectable modulation. This remains true in spectra ratioed to remove the ${ }^{14} \mathrm{~N}$ modulations, although with some attendant decrease in the signal-to-noise ratio and other uncertainties (see the Supporting Information). Moreover, because the fractional modulation depth is substantially less than that for $\mathrm{D}_{2} \mathrm{O}$ exchange (below), uncertainties in estimating the intrinsic echo amplitude and decay properties are small (dotted-dashed curve). As found by Force et al. (25), the pattern of modulation decay requires significantly inequivalent couplings for the three deuterons, with one having approximately twice the magnitude of the other two. However, Figure 8A shows that the apparent $\mathrm{D}_{3}$ $\mathrm{MeOH}$ coupling parameters inferred by Force et al. or here (Figure 6), for $200 \mathrm{~K}$ advanced samples, predict a substantially smaller overall modulation depth and somewhat slower oscillation dampening compared to those observed in flashadvanced samples (dashed curve, model 2). These modulation depth attenuation characteristics both suggest that the $\mathrm{D}_{3}$ couplings seen in flash turnover samples are indeed stronger than had previously been supposed from lowtemperature advancement studies.

Figure 8B shows an expanded version of Figure 7D, for a $\mathrm{D}_{2} \mathrm{O}$-exchanged sample in $\mathrm{S}_{2}$ with negligible shell 3 interactions. The model curves show progressively the fitted intrinsic echo amplitude, the effects of the two strongly coupled deuterons (shells 1 and 2), and the full fit (shells 1, 2 , and 4). The latter again fits the data essentially within the noise, except for the short time region where some ${ }^{14} \mathrm{~N}$ contribution is still evident. Because the deuterium modulations are deep in all $\mathrm{D}_{2} \mathrm{O}$ samples, reliable determination of the intrinsic echo amplitude function, particularly its initial value, is less direct. The fitting must be consistent with the typical magnitudes of decay envelopes containing weakly modulated signals, seen in equivalent $\mathrm{H}_{2} \mathrm{O}$-buffered samples. This results in a normalized amplitude of the first deuterium modulation cycle consistently smaller than that inferred earlier (17), with a consequent better fitting of the pattern at longer times $(>1.5 \mu \mathrm{s})$. In Figure 8B, the overall modulation depth and height of the first resolved peak are largely determined by the shell 1 and 2 contributions so that the corresponding parameter estimations, particularly the dipolar terms, are robust. The shell 4 interactions are then determined by the precise amplitude and attenuation properties of the pattern, particularly at longer times. When shell 3 interactions are absent, this latter property of the ESEEM decay is reasonably sensitive to both the number and magnitude of the shell 4 interactions. The best fit is with the values in Table 1, but combinations of an $n_{4}$ of 3 or 5 with somewhat stronger or weaker hyperfine interaction values, respectively, could not be excluded.

Figure 8C shows an expanded version of Figure 7A, for a $\mathrm{D}_{2} \mathrm{O}+\mathrm{MeOH}$ exchanged sample in $\mathrm{S}_{2}$ with maximal shell 3 interactions (as seen here). Progressive model interaction curves are also shown. Here the modulation depth is almost "total", resulting in a flattening of the general time dependence of the pattern and obscuration of the ${ }^{14} \mathrm{~N}$ contribution. However, the position of the first peak is again almost totally determined by the shell 1 and 2 parameters, which are slightly stronger than in Figure 8B. Inclusion of the shell 3 effects results in a significant further increase in modulation depth and a "first-order" fit to the data. A readily detectable refinement is brought by inclusion of the shell 4 interactions, particularly at longer times, but the pattern is now much less sensitive to these than was the case in Figure 8B. For this 
reason, the shell 4 population was locked at 4 in all simulations involving significant shell 3 effects. Although the final fit in Figure $8 \mathrm{C}$ is good, it is not within the noise, which possibly reflects the inevitably unrealistic modeling of shell 3 , with up to $\sim 10$ totally equivalent, weak couplings. Realistically, this class of deuterons would have some spread of coupling values. The range of $n_{3}$ in Table 2 is that for all the samples that were studied. For a given sample, the values of $n_{3}$ and $B_{3}$ interact in the fitting, which is effectively sensitive to their product near the optimal fit point. The uncertainty in $n_{3}$ for a given fit is less than 2 .

The consistency of the ESEEM modeling may be further tested by considering the complementary data for exchangeable proton interactions that comes from ${ }^{1} \mathrm{H}$ ENDOR studies on the $\mathrm{S}_{2}$ multiline state. ENDOR is equally sensitive to the isotropic $\left(A_{i}\right)$ and (traceless) dipolar hyperfine interactions $\left(T_{\mathrm{d} i}\right)$, but total intensities (i.e., numbers of equivalent protons in each class, $n_{i}$ ) are less reliable (40). ESEEM is most sensitive to $n_{i}$ and $T_{\mathrm{d} i}$, with the $A_{i}$ term having a more subtle effect on the modulation damping. Several $\mathrm{D}_{2} \mathrm{O}$ exchangeable proton ENDOR studies on the $\mathrm{S}_{2}$ state have been reported (17-20,22). With powder pattern samples, the only quantity normally determined is the $A_{\perp}$ turning point value (in magnitude) of the total anisotropic hyperfine coupling. For protons, this is given by

$$
A_{\perp}=A-T_{\mathrm{d}} \mathrm{MHz}
$$

The data from all groups are generally consistent and report three resolved couplings, with $A_{\perp}$ values of (i) $4.0-4.9 \mathrm{MHz}$, (ii) $2.0-2.5 \mathrm{MHz}$, and (iii) 1.0-1.2 MHz. Variable weaker couplings $(<1 \mathrm{MHz})$ are also seen. The proton ENDOR $A_{\perp}$ values corresponding to our average fitted deuteron ESEEM parameters are listed in Table 2. It is seen that classes 1, 2, and 4 fit the above ENDOR data well. The predicted coupling from class 3 is also within the range of one resolved ENDOR frequency, but it is likely that class 3 contains a range of couplings, which are more probably related to the unresolved proton background seen particularly in ESE ENDOR from the $\mathrm{S}_{2}$ multiline signal (17).

Uniqueness of Parameter Values. For a center as complex as the Mn cluster in PSII, powder pattern ESEEM data alone are unlikely to submit to a single interpretation. Generally, additional considerations are needed to suggest the most likely conclusions. With $\mathrm{DMeOH}$ binding, two parameter sets reasonably fit the data for $S_{2}$, depending on how the $S_{1}$ $\rightarrow S_{2}$ advancement occurs (flash turnover or low-temperature illumination). The inferred dipolar parameters in the former case are approximately twice those for the latter (Table 1 and Figure 6 legend). If the conservative assumption is made that no more than one $\mathrm{MeOH}$ is bound to the cluster at a given time, then the simplest interpretation, in our view, is that all such $\mathrm{MeOH}$ molecules bind equivalently. Then roughly half the multiline centers in a typical $200 \mathrm{~K}$ turnover experiment have no bound (or strongly coupled) $\mathrm{MeOH}$. The apparent heterogeneity in relaxation and other properties of the low-temperature generated multiline would be consistent with this (data presented here and in refs 24 and 27).

A somewhat similar situation occurs with the $\mathrm{D}_{2} \mathrm{O}$ exchange. The $S_{2}$ state data in Figures 5 and 7 are well fit assuming either single deuterons in shells 1 and 2, with parameters as in Table 2, or single waters (equivalent deuteron pairs) in each of these shells, with parameter values very close to those of Britt et al. (22) (not shown). The four dipolar couplings in the latter case are all quite similar and close in magnitude to the shell 2 value in Table 2, with implied $|\rho|$ values of 1 . However, as noted in ref 22, consistency with the exchangeable proton ENDOR data of PSII requires the protons on one of the above water molecules to have zero isotropic coupling to the Mn cluster, while those on the other water have isotropic couplings of $\sim 3 \mathrm{MHz}$. This is unusually high for water ligated to $\mathrm{Mn}$ (41-43). Conversely, assuming the shell occupancies and parameter values in Table 2, a consistent interpretation involving a single water molecule binding with conventional intrinsic hyperfine parameters is possible, as discussed below.

Structural Interpretation. In Gaussian units, the two hyperfine interaction parameters for each deuteron are given by

$$
T_{\mathrm{d}}=B v_{\mathrm{I}}=\left(\rho g \beta / H_{\mathrm{o}} r^{3}\right) v_{\mathrm{I}} ; \quad A=\rho A_{\text {iso }} \mathrm{MHz}
$$

$B$ is dimensionless, and $v_{\mathrm{I}}$ is the ${ }^{2} \mathrm{H}$ nuclear Zeeman frequency at the observation field $\left(H_{\mathrm{o}}\right) . A_{\text {iso }}$ is the isolated ion contact term and $\rho$ the effective electron spin projection coefficient in the point dipole approximation of the interaction of the deuteron with the Mn cluster (e.g., see refs 18 , 19 , and 41 and below). In principle, it can be different for the $A$ and $B$ terms; since the dipolar interaction is through space, it may have contributions from more than one metal center in the cluster. Generally, the $A$ term reflects only the spin projection of the metal center to which the ligand of interest is bound. In practice, the analysis is sensitive only to the magnitude of $\rho$ and $r$ is a weighted average dipolar interaction distance between the nuclear and electron spins. For a typical case where the labeled ligand binds terminally to one ion in the cluster, the cubic dependence means that $r$ is essentially the internuclear distance between that (closest) metal ion and the deuteron, while $\rho$ is the projection coefficient for the ligated metal ion. In the absence of evidence to the contrary, we adopt this simplest interpretation here. The other terms in eq 2 have their usual meanings. $g$ is $\sim 1.98$ for the $S_{2}$ multiline signal (44), and this value is also assumed for $\mathrm{S}_{0}$.

$\mathrm{DMeOH}$. From Table 1, it is apparent that the pattern and magnitude of $\mathrm{DMeOH}$ deuteron couplings, one stronger and two approximately equal and weaker, are similar in the $S_{2}$ and $\mathrm{S}_{0}$ states, with the overall interactions attenuated somewhat in $\mathrm{S}_{0}$. In Table 1 , the effective methyl deuteronMn distances for the three observed $B$ couplings are given for $|\rho|$ values of 1 and 2 for the $S_{2}$ and $S_{0}$ states. This approximately spans the magnitude range expected for the $\rho$ parameter of individual Mn ions in the cluster (see below). For both $\mathrm{S}$ states, the patterns and magnitudes of the $\mathrm{Mn}-\mathrm{D}$ distances are similar, and using a standard structure for the methoxy fragment, the ligating geometry may be inferred. For methanol binding in either $S$ state, $|\rho|$ values of significantly less than 2 for the ligating $\mathrm{Mn}$ are improbable, ${ }^{3}$

${ }^{3}$ A $10 \%$ reduction in $\rho$ to 1.8 results in an $\sim 3 \%$ reduction in the calculated $\mathrm{Mn}-\mathrm{D}$ distances in Table 1 . This yields an inferred $\mathrm{Mn}-\mathrm{O}$ distance of $1.80 \AA$, a bond length shorter than in any instance of relevant, terminal $\mathrm{Mn}-\mathrm{O}$ ligation of which we are aware. However, the calculation is influenced by the "single-ion interaction" assumption mentioned above. Its precise effect is difficult to quantify without further structural knowledge of the Mn cluster geometry. 


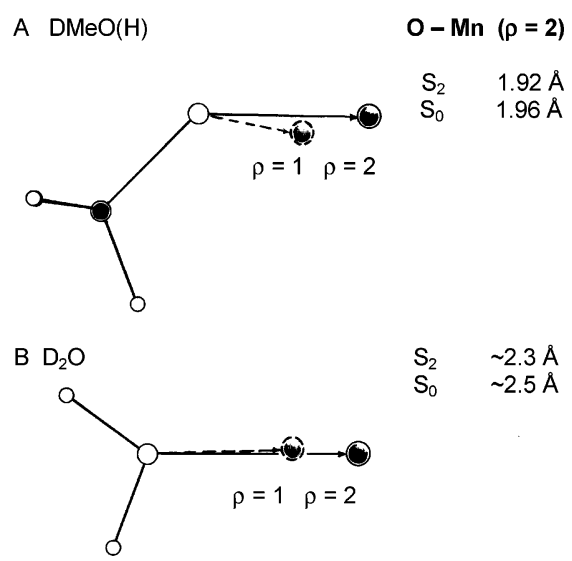

FIGURE 9: (A) Proposed methanol ligand to Mn geometries in the $\mathrm{S}_{2}$ and $\mathrm{S}_{0}$ states, based the methyl deuteron-Mn nearest neighbor dipolar distances inferred from the $B_{i}$ values in Table 1. OxygenMn separations for the assumed terminal ligating $\mathrm{Mn}$ are shown for Mn spin projection coefficient values $(\rho)$ of 1 and 2 for this $\mathrm{Mn}$ in $\mathrm{S}_{2}$ (see the text; $\rho$ values given below $\mathrm{Mn}$ positions). Only one of the four cluster $\mathrm{Mn}$ atoms is depicted here and in panel $\mathrm{B}$, in two possible positions relative to the ligating species. $S_{0}$ geometries are very similar. $\rho$ must be at least 1.8 in magnitude to give physically reasonable $\mathrm{Mn}-\mathrm{O}$ bond lengths in $\mathrm{S}_{2}$ or $\mathrm{S}_{0}$. (B) Possible close water ligand to terminal Mn geometries in the $S_{2}$ state (as in panel A), based on the oxo deuteron-Mn nearest neighbor dipolar distances inferred from the $B_{1}$ and $B_{2}$ values in Table 2 (see the text). $\mathrm{S}_{0}$ geometries are similar. Calculations assume all four atoms are in the same plane (resulting in the shortest $\mathrm{Mn}-\mathrm{O}$ distances) and $\rho \sim 2$ in both $S$ states. If $\rho \sim 1$, some deuteronMn distances would be as short as $2.3 \AA$, which is $\sim 0.4 \AA$ shorter than any previously observed distance for water ligating $\mathrm{Mn}$ (II) or $\mathrm{Mn}(\mathrm{III})$ (e.g., see refs 41-43).

due to the resulting length of the $\mathrm{Mn}-\mathrm{O}$ bond being physically unreasonable (Figure 9A and Table 1).

Even for a $|\rho|$ of 2, the inferred $\mathrm{Mn}-\mathrm{O}$ lengths are only 1.92 and $1.96 \AA$ in $S_{2}$ and $S_{0}$, respectively. We regard a $|\rho|$ of $\sim 2$ as a reliable conclusion for $S_{2}$ and $S_{0}$, due principally to the magnitudes of the methyl deuteron couplings. In fact, the average $\mathrm{S}_{2} d$-methyl coupling from Table 1 is more than $80 \%$ of that seen for $\mathrm{DMeOH}$ terminally binding to $\mathrm{Mn}(\mathrm{III})$ in a model Mn(III-IV) dimer complex, for which the Mn(III) $\rho$ value is 2.0 (41).

$\mathrm{D}_{2} \mathrm{O}$ Exchange. Following $\mathrm{D}_{2} \mathrm{O}$ exchange, all samples exhibited strong modulation in $\mathrm{S}_{2}$; however, detailed analysis shows significant differences between samples depending on both the plant species and experimental treatment (Table 2). Three classes of deuterons (classes 1,2, and 4) are seen in all samples in $S_{2}$ and in the flash samples in $S_{0}$. In the pea samples, little or no fourth class is required for fitting of the $200 \mathrm{~K}$-induced $\mathrm{S}_{2}$ state. However, in the spinach samples, a fourth class (labeled 3 ) is required. It is not clear if this is a genuine species difference or reflects the preparation procedure (Ford and Evans for peas, modified Bricker for spinach). In the $\mathrm{D}_{2} \mathrm{O}$ substitution procedure that was used, it is expected that the substrate water will exchange rapidly. However, protein matrix protons may also exchange with a slower and variable rate. The pea samples were used immediately following exchange, while the spinach samples were stored for significantly longer periods, albeit at $77 \mathrm{~K}$ during shipping, perhaps allowing more extensive exchange.

Given that all the PSII samples studied here were catalytically functional and that the minimum number of deuterons in the variable class was $\sim 0$, it seems reasonable to associate the latter with the matrix exchangeable protons. The other three classes would then represent deuterons from substrate water or hydroxyls, magnetically interacting with the Mn cluster.

From an analysis of the PSII substrate water exchange kinetics reported by Wydrzynski and co-workers (45), we have concluded that the slowly exchanging water binds terminally to a $\mathrm{Mn}(\mathrm{II})$ in $\mathrm{S}_{0}$ and to a $\mathrm{Mn}$ (III) in $\mathrm{S}_{2}(27,46)$. In addition, our recent ${ }^{17} \mathrm{O}$ ESEEM study of substrate water interaction with the $S_{2}$ multiline state (15) suggested a $|\rho|$ value of $\sim 2$ for the $\mathrm{Mn}(\mathrm{III})$ binding site and a similar ligation to that of water with the $\mathrm{Fe}^{3+}$ center in Cyt P450. When combined with the discussion above, a simple interpretation is that deuteron classes 1 and 2, each with single occupancy, correspond to one closely bound water molecule in $S_{2}$ and $\mathrm{S}_{0}$, while class 4 corresponds to approximately two second shell or otherwise more distantly bound waters.

Using ENDOR, Randall et al. (41) have determined water proton couplings in the same $\mathrm{Mn}(\mathrm{III}-\mathrm{IV})$ dimer used for the $\mathrm{DMeOH}$ studies described above. In both cases, the exchangeable ligand binds terminally, in essentially the same position, to the $\mathrm{Mn}(\mathrm{III})$ ion with a $\rho$ of 2 . The two water proton couplings were very similar, with dipolar components equivalent to a $B$ of $\sim 0.51$. The strongest dipolar interactions (classes 1 and 2) we see in $\mathrm{D}_{2} \mathrm{O}$-exchanged PSII samples are as follows: $B_{1} \sim 0.44$ and $B_{2} \sim 0.30$. Both values indicate a $\rho$ of $>1$, but their inequivalence suggests that the ligand access may be more constrained than in the model complex. One possibility, assuming $\rho=2$, is shown in Figure 9B. This gives a $\mathrm{Mn}-\mathrm{O}$ distance of $2.3-2.4 \AA$ in $\mathrm{S}_{2}$, which would be consistent with the ligand approaching along the Jahn-Teller axis of the Mn(III) (47), which has been suggested elsewhere (27). In $\mathrm{S}_{0}$, the $\mathrm{Mn}-\mathrm{O}$ bond lengthens to $\sim 2.5 \AA$ under the same assumptions. This is long for a $\mathrm{Mn}(\mathrm{II})-\mathrm{O}$ bond and may indicate that the water is loosely bridging $\mathrm{Mn}$ and another low-valent ion, possibly $\mathrm{Ca}$. The latter is known to be part of the cluster (3).

This "one-water, $|\rho| \sim 2$ " interpretation of the stronger deuteron interactions is also consistent with ${ }^{1} \mathrm{H}$ ENDOR studies on water binding to $\mathrm{Mn}$ in solvated ions and dinuclear complexes $(17-20,22)$. Assuming $\rho=2$, the $A_{1}$ and $A_{2}$ values from Table 2 correspond to single-ion proton $A_{\text {iso }}$ terms of $0.9-1.0 \mathrm{MHz}$. These are well within the range of proton isotropic couplings seen for water directly ligating $\mathrm{Mn}(\mathrm{II})$ or $\mathrm{Mn}$ (III) in various model systems; viz., $A_{\text {iso }} \sim 0.7-$ 1.3 MHz (41-43). Thus, this interpretation explains the observed $\mathrm{S}_{2}$ state proton ENDOR data in a natural way, the deuterium ESEEM and proton ENDOR coupling strengths being in the same progression, with conventional single-ion parameter values.

The $\mathrm{S}_{2}$ state ESEEM data seen here for $\mathrm{D}_{2} \mathrm{O}$-exchanged PSII are basically very similar in flash turnover or $200 \mathrm{~K}$ illumination samples. Both are comparable to data reported recently (22) for the $S_{2}$ multiline signal. This is not true, however, for the $S_{0}$ results, which here are similar to those in $S_{2}$ but quite different from those reported in the study cited above. This is presumably related to the different methods used to generate and isolate the $\mathrm{S}_{0}$ state. The flash advance and chemical trapping used in ref 22 appears to produce extensive, disordered interactions of the deuterons with the Mn cluster and a relatively poor signal-to-noise ratio in the modulation pattern. Flash turnover gives data with a 
similar signal-to-noise ratio in $\mathrm{S}_{2}$ and $\mathrm{S}_{0}$, allowing for the fact that the multiline echo amplitude in $S_{0}$ is $\sim 60-70 \%$ of that in $S_{2}$. This arises because the multiline signal in $S_{0}$ is significantly broader than in $\mathrm{S}_{2}$. However, the two strongest $\mathrm{S}_{0}$ deuteron interactions seen in ref 22 have dipolar couplings similar to those of classes 1 and 2 seen here ( 0.85 and 0.70 $\mathrm{MHz}$, vs 0.90 and $0.58 \mathrm{MHz}$ from Table 2). There appear to be more background deuterons in the chemically trapped samples. These may be related to the shell 3 effects seen here.

A surprising result from our data is that there is no additional strong deuteron coupling in $\mathrm{D}_{2} \mathrm{O} / \mathrm{MeOH}$ media, arising from the exchanged methanol -OD group. In the model complex studies of Randall et al. (41), this interaction is as strong as the deuteron couplings from water binding in the same position. For any $\rho$ value of the ligating Mn which yields a physically reasonable value for the $\mathrm{MeO}-\mathrm{Mn}$ distance, our data essentially exclude the possibility that the methoxy group is deuterated while the close water retains two deuterons. Either the methanol binds as methoxide, or when it binds as MeOD, a close deuteron (probably class 1) is lost from the substrate water, with either combination possible for $\mathrm{S}_{0}$ and $\mathrm{S}_{2}$. The short $\mathrm{MeO}-\mathrm{Mn}$ distance inferred for $S_{2}$ is certainly close to that expected for binding of oxide to Mn(III) along a non-Jahn-Teller axis (47). This effect might result from a requirement for net charge maintenance among the cluster ligands. If the neutral methanol displaces a charged protein ligand (e.g., carboxylate), then a proton must be lost (or transferred) to compensate, especially in an internal protein environment.

Finally, at least one other interpretation of the strongest deuterium couplings ( 1 and 2 ) in Table 2 is possible. They may represent hydroxyls bound to two separate Mn ions (or as bridges, e.g., ref 48), with effective $\rho$ values in the range of $\sim 1.2-2$. These hydroxyls could originate from substrate water. The close similarity of the coupling patterns in $\mathrm{S}_{0}$ and $S_{2}$ would mean that essentially the same hydroxyl binding and $\mathrm{Mn}$ spin projections occur in both $\mathrm{S}$ states. It is harder to rationalize the methanol binding results described above in this scheme, since in $\mathrm{S}_{0}$ a $\mathrm{Mn}$ (II) (see below) is then presumably binding either a methoxide or oxo species. This seems to us unlikely but cannot be excluded at present.

Implications for the Mn Cluster. The conclusion here is that both $\mathrm{MeOH}$ and one tightly bound water molecule bind to $\mathrm{Mn}$ centers in the cluster with $\rho$ values of $\sim 2$ (in magnitude). For a given net electron spin of the cluster $\left(S_{\mathrm{T}}\right.$, here $1 / 2$ ), each Mn ion has an effective spin of $\rho_{j} S_{\mathrm{T}}$, where $\rho_{j}$ is the relevant spin projection coefficient of ion $j . \rho_{j}$ values depend on the individual spins (oxidation states) of the ions, the nature of the cluster exchange coupling, and its total spin (e.g., see ref 49). At present, complete atomic structural detail of the $\mathrm{Mn} / \mathrm{Ca}$ cluster in PSII is not available, nor is there agreement about a detailed exchange coupling model for the net spin half-cluster responsible for the $\mathrm{S}_{2}$ or $\mathrm{S}_{0}$ multiline states, although several have been proposed $(48,50,51)$. However, some general considerations apply. For an antiferromagnetically coupled cluster of Mn ions in the II-IV oxidation state range [as for $S_{0}$ and $S_{2}(46,52,53)$ ], the individual projection coefficients for the lowest spin $\left(S_{\mathrm{T}}=\right.$ $1 / 2$ ) state are generally between $\sim 1$ and $\sim 2$ in magnitude, with alternating signs. In all cases so far proposed, at least one ion with the lowest oxidation state [III in $\mathrm{S}_{2}$ and II in $\mathrm{S}_{0}$
(46)] has the largest magnitude of $\rho$, which is $\sim 1.7-2.3$. Generally, only one Mn center can have a $\rho$ value this large (otherwise, the resulting multiline patterns would be too wide), so our data suggest that both $\mathrm{MeOH}$ and the tightly bound water terminally ligate the same $\mathrm{Mn}$ ion in $\mathrm{S}_{0}$ and $\mathrm{S}_{2}$. This closely bound water molecule does not displace methanol, consistent with methanol having no inhibitory effect on PSII water splitting behavior, even at solvent concentrations (5 M) well above those used here (25).

The fact that a very similar methanol binding picture emerges in $S_{0}$ and $S_{2}$ suggests that in both cases ligation occurs to a $\mathrm{Mn}$ in the lowest oxidation (i.e., highest spin) state of the cluster. In $\mathrm{S}_{0}$, only one $\mathrm{Mn}$ is in the II oxidation state (46), and this then binds methanol. The inferred $\mathrm{Mn}-\mathrm{O}$ distance is short for terminal ligation to a $\mathrm{Mn}(\mathrm{II})$ ion, so it is possible that the $\rho$ value for this ion is somewhat greater than 2. In $\mathrm{S}_{2}$, there will be at least one $\mathrm{Mn}$ (III). Given that methanol must bind in the same location in $S_{1}$ and $S_{2}$, since $200 \mathrm{~K}$ advancement produces the methanol-bound multiline form (results presented here and in ref 27), it is simplest to conclude that the same $\mathrm{Mn}$ binds the alcohol in $\mathrm{S}_{0}$ and $\mathrm{S}_{2}$. This Mn undergoes oxidation from II to III during the $\mathrm{S}_{0} \rightarrow$ $\mathrm{S}_{1}$ transition.

The location of the exchangeable deuterons in shell 3 is not obvious in terms of the current structures. The value of $B_{3}$ in Table 2 suggests minimum deuterium-Mn distances of $\sim 3-4 \AA$, assuming $|\rho|$ is in the range of $1-2$. At $3 \AA$, a deuteron is almost certainly attached to a ligand atom (here O mostly, i.e., -OD group), while at $4 \AA$, more possibilities exist. Only approximately five carboxylic side chains are currently identified as direct ligands to metals, and because of the high formal charge of the metal ions (up to +17 in $\mathrm{S}_{2}$ ), the ligating side chains and the bridge oxo's are likely unprotonated (mostly). Since $n_{3}$ can be as high as 10, it seems that neutral exchangeable groups (peptide $\mathrm{N}-\mathrm{H}$ and $\mathrm{C}-\mathrm{OH}$ side chains, etc.) must generally be involved, at distances out to $\sim 4 \AA$. Unless they are strongly variably occluded from solvent exchange, water-derived hydroxyls seem to be unlikely candidates.

The presumed second substrate water molecule may be one of the approximately two in shell 4 . These are always present and appear to have a resolved ENDOR signature. Here the closest approach distances are $\sim 3.7-4.7 \AA$, depending on $\rho$. This range is not consistent with the water being ligated to a Mn closely coupled to the EPR visible cluster in $S_{0}$ or $S_{2}$, although some bridging position in which $\rho$ cancellation occurs cannot be excluded. A binding position "more distant" from that of the close water is certainly consistent with a shell 4 water having the faster substrate exchange kinetics seen by Hillier et al. (45). Inclusion of a very weak isotropic coupling (Table 2) for these deuterons gives a better overall fit to the ESEEM and ENDOR data, suggesting some through-bond connection to the Mn cluster.

Summary. The main findings from this study regarding interactions of water and methanol with the Mn cluster in PSII are summarized below.

(i) During flash-induced functional turnover, binding of substrate water or methanol, as reflected in deuteron ESEEM couplings, is very similar in the $S_{2}$ and $S_{0}$ multiline states.

(ii) Both small molecules bind noncompetitively, probably to the same $\mathrm{Mn}$ center, in both the $\mathrm{S}_{2}$ and $\mathrm{S}_{0}$ states. 
(iii) The simplest interpretation of this binding is that it involves terminal ligation to a $\mathrm{Mn}$ in the cluster which is least oxidized in each of these $\mathrm{S}$ states. The effective spin projections for this $\mathrm{Mn}$ in either $1 / 2$ net spin state are approximately 2 in magnitude.

\section{SUPPORTING INFORMATION AVAILABLE}

Field-swept ESE spectra of flash-advanced PSII samples, $\mathrm{DMeOH} / \mathrm{MeOH}$ ratios from ESEEM experimental and modeled spectra for $\mathrm{S}_{2}$ state PSII, and one-flash - zero-flash ESEEM. This material is available free of charge via the Internet at http://pubs.acs.org.

\section{APPENDIX 1}

Echo Amplitude Decay Function. The relaxation enhancement model (35) assumes an intrinsic (background) relaxation rate which is a simple exponential and an angular-dependent dipolar relaxation interaction with some fast-relaxing paramagnet. The latter process occurs in a fraction, $F$, of total centers. In this instance, knowledge of the nature and identity of the relaxation enhancer is not required and it remains unspecified. An exact powder pattern average of the interaction is determined, with no attempt to correlate this angular dependence with that of the nuclear ESEEM interactions. Then the normalized echo decay function, $A_{\mathrm{Y}}(t)$, has the form

$$
\begin{aligned}
& A_{\mathrm{Y}}(t)= \\
& \quad F[\text { dipolar model }]+(1-F)\left[\exp \left(-k_{\text {background }} t\right)\right]
\end{aligned}
$$

The dipolar model, $I(t)$, is of the form

$$
I(t)=\int_{0}^{\pi / 2} \sin \theta\left[\mathrm{e}^{-\left(k_{\text {background }}+k_{1 \theta}\right) t}\right] \mathrm{d} \theta
$$

The angular dependence of the $k_{1 \theta}$ term was taken as

$$
k_{1 \theta}=k_{1 \mathrm{~d}}\left(1-3 \cos ^{2} \theta\right)^{2}
$$

where $k_{1 \mathrm{~d}}$ is the dipolar rate parameter, which is related to the nature of the relaxation enhancement center and the magnitude of its separation vector from the spin system of interest. $\theta$ is the angle between this separation vector and the external magnetic field.

\section{APPENDIX 2}

Deuterium ESEEM Modulation. Exact expressions for the ESEEM modulation functions for an $I=1$ nuclear spin, interacting with an $S=1 / 2$ electron spin in the limit of zero nuclear quadrupolar coupling, have been given by Dikanov et al. (34). This gives the normalized three-pulse echo modulation function, $V(\tau T)$, for a single nuclear interaction

$$
V(\tau T)={ }^{1}{ }_{2}\left[V_{\alpha}(\tau T)+V_{\beta}(\tau T)\right]
$$

where

$$
\begin{array}{r}
V_{\alpha}(\tau T)=1-16 / 3 k \sin ^{2}\left(\omega_{\beta} / 2 \tau\right) \sin ^{2}\left[\omega_{\alpha} / 2(\tau+T)\right]+ \\
16 / 3 k^{2} \sin ^{4}\left(\omega_{\beta} / 2 \tau\right) \sin ^{4}\left[\omega_{\alpha} / 2(\tau+T)\right]
\end{array}
$$

and $V_{\beta}(\tau T)$ is the equivalent expression with $\alpha$ and $\beta$ interchanged.
Here $\omega_{\text {I }}$ is the nuclear angular Zeeman frequency, which equals $g_{n} \beta_{n} H_{\mathrm{o}} / \hbar$ (radians per second) and

$$
\begin{gathered}
\omega_{\alpha}=\left[\left(A^{\prime} / 2+\omega_{\mathrm{I}}\right)^{2}+\left(B^{\prime} / 2\right)^{2}\right]^{1 / 2} \\
\omega_{\beta}=\left[\left(A^{\prime} / 2-\omega_{\mathrm{I}}\right)^{2}+\left(B^{\prime} / 2\right)^{2}\right]^{1 / 2} \\
\hbar A^{\prime}=(\rho g \beta)\left(g_{n} \beta_{n}\right) r^{-3}\left(3 \cos ^{2} \theta-1\right)+h A \\
\hbar B^{\prime}=(\rho g \beta)\left(g_{n} \beta_{n}\right) r^{-3}(3 \cos \theta \sin \theta) \\
k=\left(\omega_{\mathrm{I}} B^{\prime} / \omega_{\alpha} \omega_{\beta}\right)^{2}
\end{gathered}
$$

Equations A7 and A8 refer to the total nuclear hyperfine interaction (contact plus dipolar), where $r$ is the magnitude of the nuclear-electron spin separation vector in the point dipole approximation and $\theta$ is the angle between this vector and the external magnetic field. For each electron nuclear interaction, exact powder pattern averages of $V_{\alpha}(\tau T)$ and $V_{\beta}(\tau T)$ are determined, in a manner analogous to eq A2 given above. These are then combined according to

$$
V(\tau T)={ }^{1}{ }_{2}\left[\prod_{i} V_{\alpha i}(\tau T)^{n_{i}}+\prod_{i} V_{\beta i}(\tau T)^{n_{i}}\right]
$$

where $i$ runs over the deuteron class numbers $(1-3$ or 4$)$ and $n_{i}$ is the number of equivalent deuterons in each class.

The normalized ESEEM echo response predicted by the model is then

$$
\operatorname{ESEEM}(t)=A_{\mathrm{Y}}(t) \times V(\tau T), \text { where } t=\tau+T
$$

\section{REFERENCES}

1. Joliot, P., Barbieri, G., and Chaboud, R. (1969) Un nouveaux modele des centres photochimiques du système II, Photochem. Photobiol. 10, 309-329.

2. Kok, B., Forbush, B., and McGloin, M. (1970) Cooperation of charges in photosynthetic $\mathrm{O}_{2}$ evolution. 1: A linear four step mechanism, Photochem. Photobiol. 11, 457-475.

3. Ferreira, K. N., Iverson, T. M., Maghlaoui, K., Barber, J., and Iwata, S. (2004) Architecture of the Photosynthetic OxygenEvolving Center, Science 303, 1831-1838.

4. Biesiadka, J., Loll, B., Kern, J., Irrgang, K.-D., and Zouni, A. (2004) Crystal structure of cyanobacterial photosystem II at 3.2 A resolution: A closer look at the Mn-cluster, Phys. Chem. Chem. Phys. 6, 4733-4736.

5. Kamiya, N., and Shen, J.-R. (2003) Crystal structure of oxygenevolving photosystem II from Thermosynechococcus vulcanus at 3.7 A resolution, Proc. Natl. Acad. Sci. U.S.A. 100, 98-103.

6. Nugent, J. H. A. (2001) Photosynthetic water Oxidation: Special Edition, Biochim. Biophys. Acta 1503, 1-259.

7. Yachandra, V. K., DeRose, V. J., Latimer, M. J., Mukerji, I., Sauer, K., and Klein, M. P. (1993) Where plants make oxygen: A structural model for the photosynthetic oxygen-evolving manganese cluster, Science 260, 675-679.

8. MacLachlan, D. J., Nugent, J. H. A., and Evans, M. C. W. (1994) A XANES study of the manganese complex of inhibited PS II membranes indicates manganese redox changes between the modified $\mathrm{S}_{1}, \mathrm{~S}_{2}$ and $\mathrm{S}_{3}$ states, Biochim. Biophys. Acta 1185, 103111.

9. Messinger, J., Nugent, J. H. A., and Evans, M. C. W. (1997) Detection of an EPR multiline signal for the $\mathrm{S}_{0} *$ state in photosystem II, Biochemistry 36, 11055-11060.

10. Ahrling, K. A., Peterson, S., and Styring, S. (1997) An Oscillating Manganese Electron Paramagnetic Resonance Signal from the $S_{0}$ State of the Oxygen Evolving Complex in Photosystem II, Biochemistry 36, 13148-13152. 
11. Dismukes, G. C., and Siderer, Y. (1981) Intermediates of a polynuclear manganese center involved in photosynthetic oxidation of water, Proc. Natl. Acad. Sci. U.S.A. 78, 274-278.

12. Dexheimer, S. L., and Klein, M. P. (1992) Detection of a paramagnetic intermediate in the photosynthetic oxygen-evolving complex, J. Am. Chem. Soc. 114, 2821-2826.

13. Matsukawa, T., Mino, H., Yoneda, D., and Kawamori, A. (1999) Dual-mode EPR study of new signals from the S3-state of the oxygen-evolving complex in photosystem II, Biochemistry 38, 4072-4077.

14. Campbell, K. A., Peloquin, J. M., Pham, D. P., Debus, R. J., and Britt, R. D. (1998) Parallel polarization EPR detection of an $S_{1}$ state 'multiline' EPR signal in photosystem II particles from Synechocystis sp. PCC 6803, J. Am. Chem. Soc. 120, 447-448.

15. Evans, M. C. W., Nugent, J. H. A., Ball, R. J., Muhiuddin, I., and Pace, R. J. (2004) Evidence for a Direct Manganese-Oxygen Ligand in Water Binding to the $\mathrm{S}_{2}$ State of the Photosynthetic Water Oxidation Complex, Biochemistry 43, 989-994.

16. Thomann, H., Bernardo, M., Goldfarb, D., Kroneck, P. M. N., and Ullrich, V. (1995) Evidence for water binding to the Fe center in cytochrome $\mathrm{P} 450$ cam obtained by ${ }^{17} \mathrm{O}$ electron spin-echo envelope modulation spectroscopy, J. Am. Chem. Soc. 117, 82438251.

17. Aznar, C. P., and Britt, D. R. (2002) Simulations of the ${ }^{1} \mathrm{H}$ electron spin-echo-electron nuclear double resonance and ${ }^{2} \mathrm{H}$ electron spin-echo envelope modulation spectra of exchangeable hydrogen nuclei coupled to the $\mathrm{S}_{2}$-state photosystem II manganese cluster, Philos. Trans. R. Soc. London, Ser. B 357, 1359-1366.

18. Fiege, R., Zweygart, W., Bittl, R., Adir, N., Renger, G., and Lubitz, W. (1996) EPR and ENDOR studies of the water oxidizing complex of photosystem II, Photosynth. Res. 48, 227-237.

19. Tang, X.-S., Sivaraja, M., and Dismukes, G. C. (1993) Protein and substrate coordination to the manganese cluster in the photosynthetic water oxidizing complex: ${ }^{15} \mathrm{~N}$ and ${ }^{1} \mathrm{H}$ ENDOR spectroscopy of the $S_{2}$ state multiline signal in the thermophilic cyanobacterium Synechococcus elongatus, J. Am. Chem. Soc. 115, 2382-2389.

20. Kawamori, A., Inui, T., Ono, T.-A., and Inoue, Y. (1989) ENDOR study on the position of hydrogens close to the manganese cluster in $\mathrm{S}_{2}$ state of photosystem II, FEBS Lett. 254, 291-224.

21. Evans, M. C. W., Rich, A. M., and Nugent, J. H. A. (2000) Evidence for the presence of a component of the Mn complex of the photosystem II reaction centre which is exposed to water in the S2 state of the water oxidation complex, FEBS Lett. 477, 113117.

22. Britt, D. R., Campbell, K. A., Peloquin, J. F., Gilchrist, M. L., Aznar, C. P., Dicus, M. M., Robblee, J. H., and Messinger, J. (2004) Recent pulsed EPR studies of the Photosystem II oxygenevolving complex: Implications as to water oxidation mechanisms, Biochim Biophys. Acta 1655, 158-171.

23. Britt, R. D., Zimmermann, J.-L., Sauer, K., and Klein, M. P. (1989) Ammonia binds to the catalytic Mn of the oxygen-evolving complex of photosystem II: Evidence by electron spin-echo envelope modulation spectroscopy, J. Am. Chem. Soc. 111, 35223532.

24. Evans, M. C. W., Gourovskaya, K., and Nugent, J. H. A. (1999) Investigation of the interaction of the water oxidising manganese complex of photosystem II with the agueuous solvent environment, FEBS Lett. 450, 285-288.

25. Force, D. A., Randall, D. W., Lorigan, G. A., Clemens, K. L., and Britt, R. D. (1998) ESEEM studies of alcohol binding to the manganese cluster of the oxygen evolving complex of photosystem II, J. Am. Chem. Soc. 120, 13321-13333.

26. Clemens, K. L., Force, D. A., and Britt, D. R. (2002) Acetate Binding at the Photosystem II Oxygen Evolving Complex: An $\mathrm{S}_{2}$-State Multiline Signal ESEEM Study, J. Am. Chem. Soc. 124, 10921-10933.

27. Åhrling, K. A., Evans, M. C. W., Nugent, J. H. A., and Pace, R. (2004) The two forms of the $S_{2}$ state multiline signal in Photosystem II: Effect of methanol and ethanol, Biochim. Biophys. Acta 1656, 66-77.

28. Messinger, J., Hillier, W., and Wydrzynski, T. (1998) Kinetic Determination of the Fast Exchanging Substrate Water Molecule in the $\mathrm{S}_{3}$ State of Photosystem II, Biochemistry 37, 16908-16914.

29. Hillier, W., and Wydrzynski, T. (2000) The affinities for the two substrate water binding sites in the $\mathrm{O}_{2}$ evolving complex of photosystem II vary independently during S-state turnover, Biochemistry 39, 4399-4405.
30. Messinger, J., Robblee, J., Yu, W. O., Sauer, K., Yachandra, V. K., and Klein, M. P. (1997) The $S_{0}$ state of the oxygen evolving complex in photosystem II is paramagnetic: Detection of an EPR multiline signal, J. Am. Chem. Soc. 119, 11349-11350.

31. Yamauchi, T., Mino, H., Matsukawa, T., Kawamori, A., and Ono, T. (1997) Parallel polarization electron paramagnetic resonance studies of the $\mathrm{S}_{1}$-state manganese cluster in the photosynthetic oxygen evolving system, Biochemistry 36, 7520-7526.

32. Ford, R. C., and Evans, M. C. W. (1983) Isolation of a photosystem 2 preparation from higher plants with highly enriched oxygen evolution activity, FEBS Lett. 160, 159-164.

33. Porra, R. J., Thompson, W. A., and Kriedermann, P. E. (1989) Determination of accurate extinction coefficients and simultaneous equations for assaying chlorophylls $a$ and $b$ extracted with four different solvents: Verification of the concentration of chlorophyll standards by atomic absorption spectroscopy, Biochim. Biophys. Acta 975, 384-394.

34. Dikanov, S. A., and Tsvetkov, Y. D. (1992) Electron Spin-Echo Envelope Modulation (ESEEM) Spectroscopy, CRC Press, Boca Raton, FL.

35. Mamedov, F., Smith, P. J., Styring, S., and Pace, R. (2004) Relaxation behaviour of the tyrosine $Y_{D}$ radical in photosystem II: Evidence for strong dipolar interaction with paramagnetic centers in the $\mathrm{S}_{1}$ and $\mathrm{S}_{2}$ states, Phys. Chem. Chem. Phys. 4, 48904896.

36. Narayana, M., Zhan, R. Y., and Kevan, L. (1985) ESR and electron spin-echo modulation spectroscopic studies of molybdenumadsorbate interactions on supported molybdenum/silica, J. Phys. Chem. 89, 636-641.

37. Mims, W. B., and Peisach, J. (1981) in Biological Magnetic Resonance (Berliner, L. J., and Reuben, J., Eds.) pp 213-263, Plenum, New York.

38. Tang, X.-S., Diner, B. A., Larsen, B. S., Gilchrist, M. L., Jr., Lorigan, G. A., and Britt, R. D. (1994) Identification of histidine at the catalytic site of the photosynthetic oxygen-evolving complex, Proc. Natl. Acad. Sci. U.S.A. 91, 704-708.

39. Debus, R. J., Campbell, K. A., Gregor, W., Li, Z.-L., Burnap, R. L., and Britt, R. D. (2001) Does histidine 332 of the D1 polypeptide ligate the manganese cluster in photosystem II? An electron spin-echo envelope modulation study, Biochemistry 40, 3690-3699.

40. Reijerse, E. J., Van Aerle, N. A. J. M., Keijzers, C. P., Bottcher, R., Kirmse, R., and Stach, J. (1986) Comparison of ESEEM, ESEENDOR and CW-ENDOR on ${ }^{14} \mathrm{~N}$ in a powder, J. Magn. Reson. 67, 114-124.

41. Randall, D. W., Gelasco, A., Caudle, M. T., Pecoraro, V. L., and Britt, R. D. (1997) ESE-ENDOR and ESEEM characterization of water and methanol ligation to a dinuclear Mn(III)Mn(IV) complex, J. Am. Chem. Soc. 119, 4481-4491.

42. Sivaraja, M., Stouch, T. R., and Dismukes, G. C. (1992) Solvent Structure around Cations Determined by ${ }^{1} \mathrm{H}$ ENDOR Spectroscopy and Molecular Dynamics Simulation, J. Am. Chem. Soc. 114, 9600-9603.

43. Tan, X., Bernardo, M., Thomann, H., and Scholes, C. P. (1993) Pulsed and continuous wave electron nuclear double resonance patterns of aquo protons coordinated in frozen solution to high spin $\mathrm{MN}^{2+}$, J. Chem. Phys. 98, 5147-5157.

44. Smith, P. J., Ahrling, K. A., and Pace, R. J. (1993) Nature of the $\mathrm{S}_{2}$ state electron paramagnetic resonance signals from the oxygen evolving complex of photosystem II: Q-band and oriented X-band studies, J. Chem. Soc., Faraday Trans. 89, 2863-2868.

45. Hillier, W., and Wydrzynski, T. (2001) Oxygen ligand exchange at metal sites: Implications for the $\mathrm{O}_{2}$ evolving mechanism of photosystem II, Biochim. Biophys. Acta 1503, 197-209.

46. Kuzek, D., and Pace, R. J. (2001) Probing the Mn oxidation states in the OEC. Insightss from spectroscopic, computational and kinetic data, Biochim. Biophys. Acta 1503, 123-137.

47. Mikuriya, M., Yamato, Y., and Tokii, T. (1992) 1,3-Bis(salicylideneamino)-2-propanol as the Ligand for Manganese(III) Ions, Bull. Chem. Soc. Jpn. 65, 1466-1468.

48. Carrell, T. G., Tyryshkin, A. M., and Dismukes, G. C. (2002) An evaluation of structural models for the photosynthetic wateroxidizing complex derived from spectroscopic and X-ray diffraction signatures, J. Biol. Inorg. Chem. 7, 2-22.

49. Bencini, A., and Gatteschi, D. (1990) Electron Paramagnetic Resonance of Exchange Coupled Systems, 1st ed., Springer-Verlag, Berlin. 
50. Peloquin, J. M., Campbell, K. A., Randall, D. W., Evanchik, M. A., Pecoraro, V. L., Armstrong, W. H., and Britt, D. R. (2000) ${ }^{55} \mathrm{Mn}$ pulsed ENDOR of the S2-state multiline EPR signal of photosytem II: Implications on the structure of the tetranuclear Mn cluster, J. Am. Chem. Soc. 122, 10926-10942.

51. Pace, R. J., and Åhrling, K. A. (2004) Water oxidation in PSII: $\mathrm{H}$ atom abstraction revisited, Biochim. Biophys. Acta 1655, 172178.
52. Yachandra, V. K., Sauer, K., and Klein, M. P. (1996) Manganese cluster in photosynthesis: Where plants oxidise water to dioxygen, Chem. Rev. 96, 2927-2950.

53. Dau, H., Iuzzolino, L., and Dittmer, J. (2001) The tetra-manganese complex of photosystem II during its redox cycle: X-ray absorption results and mechanistic implications, Biochim. Biophys. Acta 1503, 24-39.

BI052146M 